\title{
The chemical composition of metal-poor emission-line galaxies in the Data Release 3 of the Sloan Digital Sky Survey $\star$
}

\author{
Y. I. Izotov ${ }^{1}$, G. Stasińska ${ }^{2}$, G. Meynet ${ }^{3}$, N. G. Guseva ${ }^{1}$, and T. X. Thuan ${ }^{4}$ \\ 1 Main Astronomical Observatory, Ukrainian National Academy of Sciences, Zabolotnoho 27, Kyiv 03680, Ukraine \\ e-mail: izotov@mao.kiev.ua \\ 2 LUTH, Observatoire de Meudon, 92195 Meudon Cedex, France \\ 3 Geneva Observatory, 1290 Sauverny, Switzerland \\ 4 Astronomy Department, University of Virginia, Charlottesville, VA 22903, USA
}

Received 5 July 2005 / Accepted 15 November 2005

\section{ABSTRACT}

We have re-evaluated empirical expressions for the abundance determination of $\mathrm{N}, \mathrm{O}, \mathrm{Ne}, \mathrm{S}, \mathrm{Cl}, \mathrm{Ar}$ and $\mathrm{Fe}$ taking into account the latest atomic data and constructing an appropriate grid of photoionization models with state-of-the art model atmospheres. Using these expressions we have derived heavy element abundances in the $\sim 310$ emission-line galaxies from the Data Release 3 of the Sloan Digital Sky Survey (SDSS) with an observed $\mathrm{H} \beta$ flux $F(\mathrm{H} \beta)>10^{-14} \mathrm{erg} \mathrm{s}^{-1} \mathrm{~cm}^{-2}$ and for which the [O III] $\lambda 4363$ emission line was detected at least at a $2 \sigma$ level, allowing abundance determination by direct methods. The oxygen abundance $12+\log \mathrm{O} / \mathrm{H}$ of the SDSS galaxies lies in the range from $\sim 7.1\left(Z_{\odot} / 30\right)$ to $\sim 8.5\left(0.7 Z_{\odot}\right)$. The SDSS sample is merged with a sample of 109 blue compact dwarf (BCD) galaxies with high quality spectra, which contains extremely low-metallicity objects. We use the merged sample to study the abundance patterns of low-metallicity emission-line galaxies. We find that extremely metal-poor galaxies $\left(12+\log \mathrm{O} / \mathrm{H}<7.6\right.$, i.e. $\left.Z<Z_{\odot} / 12\right)$ are rare in the SDSS sample. The $\alpha$ element-to-oxygen abundance ratios do not show any significant trends with oxygen abundance, in agreement with previous studies, except for a slight increase of $\mathrm{Ne} / \mathrm{O}$ with increasing metallicity, which we interpret as due to a moderate depletion of $\mathrm{O}$ onto grains in the most metal-rich galaxies. The Fe/O abundance ratio is smaller than the solar value, by up to 1 dex at the high metallicity end. We also find that $\mathrm{Fe} / \mathrm{O}$ increases with decreasing $\mathrm{H} \beta$ equivalent width $E W(\mathrm{H} \beta)$. We interpret this as a sign of strong depletion onto dust grains, and gradual destruction of those grains on a time scale of a few Myr. All the galaxies are found to have $\log \mathrm{N} / \mathrm{O}>-1.6$, implying that they have a different nature than the subsample of high-redshift damped Ly $\alpha$ systems with $\log \mathrm{N} / \mathrm{O}$ of $\sim-2.3$ and that their ages are larger than 100-300 Myr. We confirm the apparent increase in N/O with decreasing $E W(\mathrm{H} \beta)$, already shown in previous studies, and explain it as the signature of gradual nitrogen ejection by massive stars from the most recent starburst.

Key words. galaxies: ISM - galaxies: starburst - galaxies: abundances

\section{Introduction}

Knowledge of the detailed chemical composition of galaxies is fundamental to our understanding of stellar nucleosynthesis and evolution of galaxies. Emission-line galaxies provide an easy way to determine the abundances of such elements as He, $\mathrm{N}, \mathrm{O}, \mathrm{Ne}, \mathrm{S}, \mathrm{Cl}, \mathrm{Ar}$ and $\mathrm{Fe}$, out to redshifts of about 0.4 , from an analysis of the radiation from their $\mathrm{H}$ II regions in the visible domain. These determinations are considered more reliable if the electron temperatures can be measured directly, using the [O III] $\lambda 4363 /[\mathrm{O}$ III] $\lambda 5007$ line ratio. Then, the ionic abundances can be derived directly from the strengths of the emission lines. The assumptions to derive elemental abundances

* Full Tables 1 and 2 are only available in electronic form at the CDS via anonymous ftp to

cdsarc.u-strasbg.fr (130.79.128.5) or via

http://cdsweb.u-strasbg.fr/cgi-bin/qcat?J/A+A/448/955 are reduced to a minimum. The weak [O III] $\lambda 4363$ can only be measured in metal-poor emission-line galaxies, where the $\mathrm{H}$ II regions suffer little cooling and are at high enough temperature to produce significant emission of this line.

Metal-poor galaxies are the least chemically evolved galaxies and thus provide the simplest test beds for theories of chemical evolution of galaxies. They also possibly constitute a local counterpart to primeval high-redshift galaxies. In this spirit, they have been the subject of many studies in the recent past (e.g., Izotov \& Thuan 2004b, and references therein). They have also been used for the quest of the abundance of primordial helium (e.g. Izotov \& Thuan 2004a, and references therein). Those studies were based on limited samples of very different coverages and selection criteria.

The Sloan Digital Sky Survey (SDSS, York et al. 2000) offers a gigantic data base of galaxies with well-defined selection criteria and observed in a homogeneous way. In addition, the 
spectral resolution is much better than that of most previous data bases on emission-line galaxies. From this data base, it is possible to extract a sample of emission-line galaxies with well defined criteria.

Two papers have already used the SDSS to find objects with detected [O III] 24363 . The first one is by Izotov et al. (2004), and was based on the Early Data Release. It studied the abundance patterns of metal-poor emission-line galaxies, with emphasis on the N/O ratio. The second one is by Kniazev et al. (2004), who produced a catalogue of emission-line galaxies with low oxygen abundances from the Data Release 1. In both cases, the abundances were computed using the formulae given by Izotov et al. (1994).

Here, we use the Data Release 3 (DR3). We adopted selection criteria different from those by Izotov et al. (2004) and Kniazev et al. (2004) so that our sample is in general composed of objects with higher quality spectra and therefore more reliable abundances. In the present paper we compute the abundances with revised expressions, based on the most recent atomic coefficients for the line emissivities and on appropriate series of photoionization models for the ionization correction factors. All the results are available in electronic tables. We believe that they should provide a useful basis for many future works dealing with such objects.

The organization of the paper is as follows. The selection criteria used to extract the objects and the resulting sample are described in Sect. 2. The derivation of the elemental abundances is presented in Sect. 3. The obtained abundance patterns are discussed in Sect. 4. The main conclusions of this study are presented in Sect. 5.

\section{The samples}

\subsection{The SDSS DR3 data}

The SDSS DR3 (Abazajian et al. 2005) provides spectra in the wavelength range $\sim 3800-9300 \AA$ of $\sim 530000$ galaxies, quasars and stars selected over 4188 square degrees, and tables of measured parameters from these data. We have extracted the flux-calibrated spectra of the SDSS DR3 galaxies from the SDSS web page with the address http://www. sdss. org/dr3.

From a visual examination of all the spectra and subsequent measurements of emission line fluxes, we extracted $\sim 2700$ spectra with an [O III] $\lambda 4363$ emission line detected at a level better than $1 \sigma$. High-excitation Sy2 galaxies with strong [O III] $\lambda 4363$ emission line can be recognized because of their strong [O II] $\lambda 3727$, He II $\lambda 4686$, [O III] $\lambda 5007$ and [N II] $\lambda 6583$ emission lines, and have been removed from the sample. The reduction of the spectra and the measurement of the line intensities is done following the prescriptions of Izotov et al. (2004). In particular, using the IRAF/SPLOT routine, the line intensities are fitted with a Gaussian profile, and the continuum is visually defined on both sides of each line.

In the case of hydrogen lines, where the nebular emission line sits on top of a much broader stellar absorption line, the level of the continuum is set at the bottom of the absorption feature. Given the spectral resolution of the SDSS, this absorption feature is clearly seen whenever it is important. We estimate that, for the objects studied in this paper, which have large equivalent widths of the hydrogen emission lines, the presence of this underlying absorption introduces only a small uncertainty $(<10 \%)$ on the fluxes of the strong hydrogen emission lines.

The 3800-9300 A wavelength coverage of the SDSS spectra introduces two sources of uncertainties in the emission line fluxes. First, the emission line [O II] $\lambda 3727$ in the spectra of galaxies with $z \lesssim 0.02$ is not observed. The fraction of such galaxies is $\sim 30 \%$. In those cases the flux of the [O II] $\lambda 3727$ emission line is estimated from the fluxes of the [O II] 77320,7330 emission lines taking into account the empirical relation between the [O II] and [O III] temperatures (see Eq. (14)). Kniazev et al. (2004) have shown that the use of the [O II] $\lambda 7320,7330$ emission lines instead of the [O II] $\lambda 3727$ emission does not introduce biases in the derived oxygen abundances. Therefore we use the former lines to derive the $\mathrm{O}^{+}$abundance in the $\mathrm{H}$ II regions with the non-detected [O II] $\lambda 3727$ emission line. Second, differential refraction effects may be important. The majority of spectra in our sample have been obtained at airmasses $\lesssim 1.3$. For an airmass of 1.3 , the offset of the $\mathrm{H}$ II region (or of a standard star) at wavelengths $4000 \AA$ and $7500 \AA$ relative to its nominal location inside the slit at $5000 \AA$ are $+0{ }^{\prime} 5$ and $-0,5$, respectively (Fillipenko 1982). For comparison, the radius of the round slit used in the spectroscopic observations is $1 . " 5$. Therefore, the observed fluxes of the blue lines [O II] $\lambda 3727$ and [Ne III] $\lambda 3868$ and the red lines [Ar III] $\lambda 7135$ and [O II] $\lambda 7320,7330$ used for abundance determination could be affected by differential refraction. From existing data we cannot estimate the uncertainties introduced by this effect. Fortunately, strong hydrogen emission lines in the wavelength range $\sim 3700-6600 \AA \AA$ are present in the spectra of the majority of the selected galaxies. Correction for extinction using the observed fluxes of these lines also corrects at the same time for the effects of differential refraction.

The precision of abundance determinations is critically dependent on the uncertainties in the measurements of the [O III] $\lambda 4363$ emission line fluxes. In the case of galaxies with weak [O III] $\lambda 4363$ emission, observational uncertainties (Izotov et al. 2004) may create spurious trends in the abundance patterns. Therefore, we restricted our initial sample of $2700 \mathrm{ob}-$ jects to objects with the best derived abundances. Empirically, we find that we can significantly reduce unphysical trends when restricting the sample to objects with an observed flux in the $\mathrm{H} \beta$ emission line larger than $10^{-14} \mathrm{erg} \mathrm{s}^{-1} \mathrm{~cm}^{-2}$. Additionally, we have excluded all galaxies with both [O III] $\lambda 4959 / \mathrm{H} \beta<0.7$ and $[\mathrm{O}$ II $] \lambda 3727 / \mathrm{H} \beta>1.0$. A weak [O III] $\lambda 4363$ line in these galaxies may be subject to significant enhancement due to a mechanism different from photoionization heating in $\mathrm{H}$ II regions (Izotov et al. 2004) ${ }^{1}$. Applying all these selection criteria

\footnotetext{
1 The second condition [O II] $\lambda 3727 / \mathrm{H} \beta>1.0$ ensures that, by removing objects with weak [O III] $\lambda 4959$ we do not remove at the same time the most metal-deficient high-excitation H II regions. For example, both the SE and NW components of I $\mathrm{Zw} 18$ have $[\mathrm{O}$ III] $\lambda 4959 / \mathrm{H} \beta<0.7$, but also weak [O II] $\lambda 3727 / \mathrm{H} \beta$. Therefore, they are not removed from the sample.
} 
Table 1. Emission line fluxes in the SDSS galaxies ${ }^{1}$.

\begin{tabular}{|c|c|c|c|c|c|c|c|c|c|c|c|c|c|}
\hline Spectrum & [NeIII] & [OIII] & [FeIII] & [OIII] & $\mathrm{He} \mathrm{I}$ & {$[\mathrm{O} \mathrm{I}]$} & [SIII] & {$[\mathrm{NII}]$} & {$[\mathrm{SII}]$} & [ArIII] & $E W$ & $F^{2}$ & $C(\mathrm{H} \beta)$ \\
\hline number & 3869 & 4363 & 4658 & 4959 & 5876 & 6300 & 6312 & 6584 & 6725 & 7135 & $\AA$ & & \\
\hline \multirow[t]{2}{*}{$0266-51630-100^{3}$} & 0.370 & 0.116 & 0.005 & 1.911 & 0.117 & 0.024 & 0.017 & 0.036 & 0.142 & 0.055 & 262 & 502 & 0.00 \\
\hline & 0.012 & 0.005 & 0.002 & 0.057 & 0.004 & 0.002 & 0.001 & 0.002 & 0.004 & 0.003 & & & \\
\hline \multirow[t]{2}{*}{$0267-51608-384^{3}$} & 0.444 & 0.042 & 0.010 & 1.263 & 0.088 & 0.020 & 0.010 & 0.096 & 0.219 & 0.053 & 57 & 314 & 0.19 \\
\hline & 0.018 & 0.006 & 0.004 & 0.042 & 0.005 & 0.003 & 0.003 & 0.005 & 0.008 & 0.003 & & & \\
\hline \multirow[t]{2}{*}{$0270-51909-306^{3}$} & 0.175 & 0.015 & $\ldots$ & 0.967 & 0.130 & 0.026 & 0.011 & 0.294 & 0.518 & $\ldots$ & 55 & 104 & 0.10 \\
\hline & 0.014 & 0.009 & $\ldots$ & 0.037 & 0.010 & 0.006 & 0.006 & 0.015 & 0.019 & $\ldots$ & & & \\
\hline \multirow[t]{2}{*}{$0272-51941-365^{3}$} & 0.297 & 0.023 & 0.006 & 1.131 & 0.112 & 0.058 & 0.023 & 0.224 & 0.502 & 0.076 & 31 & 110 & 0.13 \\
\hline & 0.019 & 0.012 & 0.011 & 0.042 & 0.010 & 0.009 & 0.008 & 0.013 & 0.020 & 0.008 & & & \\
\hline \multirow[t]{2}{*}{$0275-51910-429^{3}$} & 0.430 & 0.094 & $\ldots$ & 1.587 & 0.112 & 0.029 & 0.012 & 0.059 & 0.237 & 0.056 & 107 & 197 & 0.03 \\
\hline & 0.017 & 0.007 & $\ldots$ & 0.051 & 0.006 & 0.004 & 0.003 & 0.004 & 0.009 & 0.004 & & & \\
\hline
\end{tabular}

${ }^{1}$ Only the first five galaxies are shown here. The total list of galaxies is available in electronic form at the CDS. The hydrogen, [O II] $\lambda 3727$,

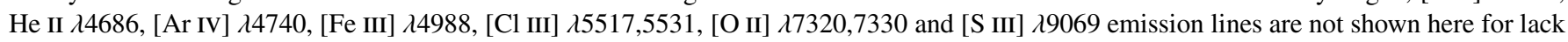
of space, but they are present in the electronic table.

${ }^{2}$ In units $10^{-16} \mathrm{erg} \mathrm{s}^{-1} \mathrm{~cm}^{-2}$.

${ }^{3}$ The first row are values, the second row are errors.

gives us a sample of $~ 310$ SDSS objects, consisting mainly of high-excitation H II regions. The [O III] $\lambda 4363$ emission line in these galaxies is detected at the $\gtrsim 2 \sigma$ level for all of them and at the $\gtrsim 5 \sigma$ for half of them.

The observed line fluxes $F(\lambda)$ are measured using the IRAF/SPLOT routine. The errors in the line fluxes are calculated from the photon statistics. For this we use files attached to each spectrum which are generated by the pipeline data reduction and which contain the error in each pixel. Additionally, we adopt an error of $2 \%$ in the emission line fluxes to account for the uncertainties of standard star absolute fluxes used for flux calibration (e.g., Oke 1990). These errors will be later propagated into the calculation of abundance errors. The line fluxes were corrected for both reddening (Whitford 1958) and underlying hydrogen stellar absorption derived simultaneously by an iterative procedure as described in Izotov et al. (1994). This procedure automatically corrects emission line fluxes for differential refraction effects. The spectrum plate number in the SDSS database, the extinction-corrected emission line fluxes $I(\lambda)$ normalized to $I(\mathrm{H} \beta)$, together with their uncertainties, the equivalent width $E W$, the observed flux $F$ of the $\mathrm{H} \beta$ emission line and the extinction coefficient $C(\mathrm{H} \beta)$ are shown in Table 1.

Figure 1 shows the location of the galaxies of the SDSS sample in the [O III] $\lambda 5007 / \mathrm{H} \beta$ vs. [N II] $\lambda 6583 / \mathrm{H} \alpha$ diagram which is generally used to distinguish H II galaxies ionized only by massive stars from galaxies containing an active nucleus (AGN). The dashed line separates the two classes of galaxies (Kauffmann et al. 2003). As in Izotov et al. (2004), all but one of our selected objects fall in the "H II" region of the diagram.

\subsection{The HeBCD sample}

In addition to the SDSS sample, we also use a sample of galaxies which has been collected primarily to study the helium abundances in low-metallicity blue compact dwarf galaxies (the HeBCD sample). This sample is the same as in

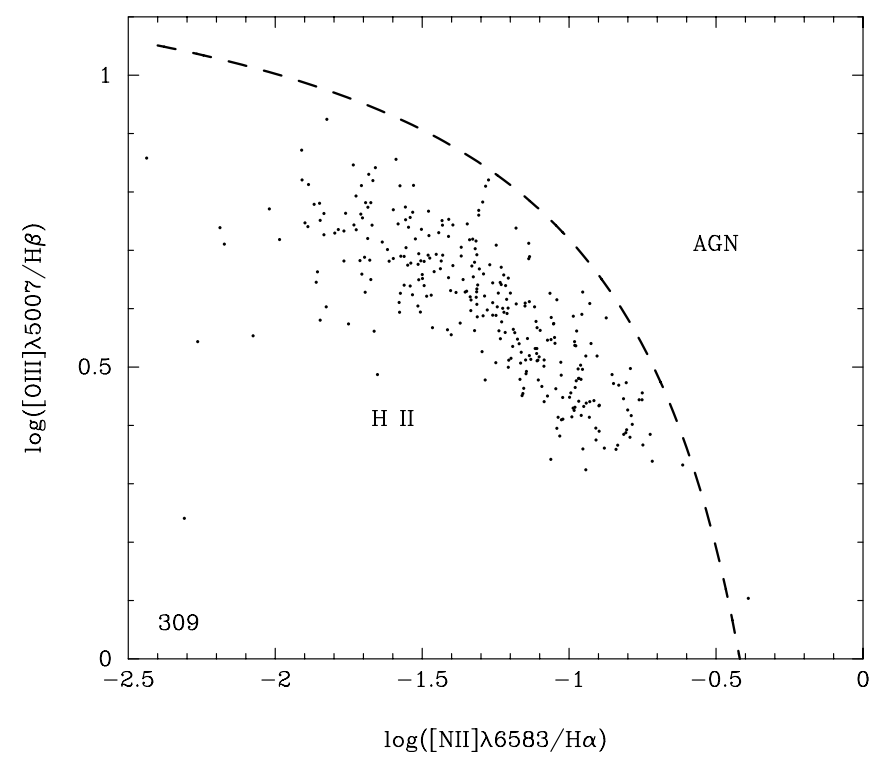

Fig. 1. Diagnostic diagram [O III] $\lambda 5007 / \mathrm{H} \beta$ vs. [N II] $\lambda 6583 / \mathrm{H} \alpha$ for our SDSS sample. The dashed line separates galaxies with $\mathrm{H}$ II regions-like spectra from galaxies containing an active nucleus (labeled "AGN") (Kauffmann et al. 2003). As in all the figures with observational data, the number of points is indicated in the left lower corner.

Izotov et al. (2004), with the addition of galaxies from Izotov \& Thuan (2004a).

\section{Element abundance determination}

The physical conditions and element abundances are derived from the reddening-corrected emission line fluxes using the electron temperature derived from the [O III] $\lambda 4363 /(\lambda 4959+\lambda 5007)$ ratio. Temperature corrections to derive ionic abundances and ionization corrections to derive elemental abundances are based on sequences of 


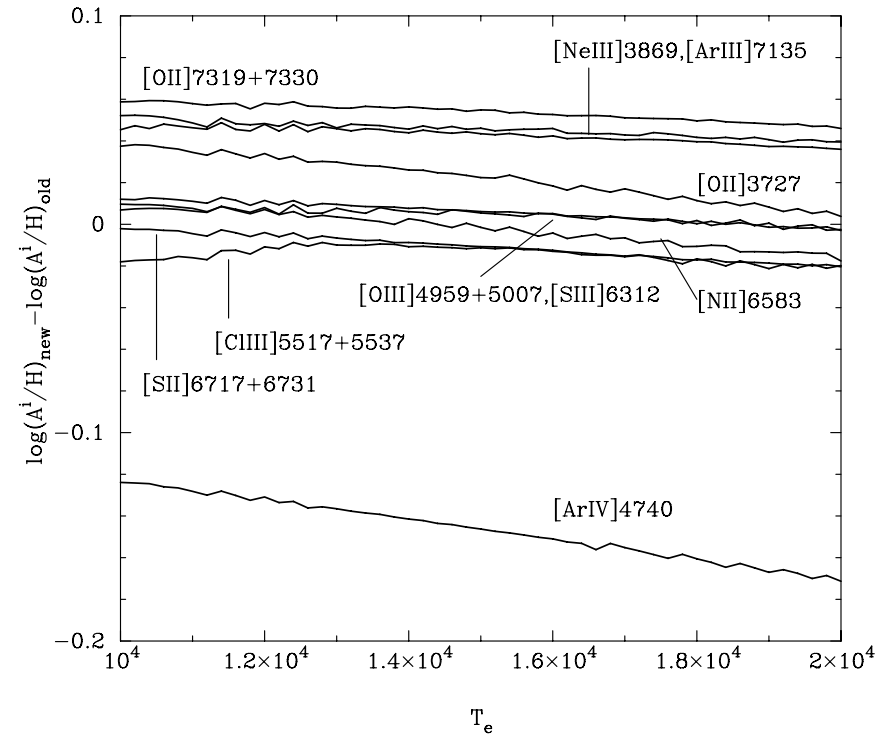

Fig. 2. Difference between ionic abundances calculated with the new and old emissivities in the case of the low-density limit as a function of the electron temperature. Curves are labeled by the emission lines used for the abundance determination.

photoionization models that best reproduce the observed emission line trends in $\mathrm{H}$ II galaxies (Stasińska \& Izotov 2003).

\subsection{Atomic data and ionic abundances}

In previous studies, we have used emissivities for emission lines provided by Aller (1984). Since that time the collisional strengths for many transitions have been re-evaluated. Here, we use the atomic data from the references listed in Stasińska (2005).

The electron temperatures derived from the [O III] $] \lambda 4363 / \lambda 5007$ line ratio using old and new emissivities differ by less than $1 \%$ in the temperature range 5000-20000 K. Therefore, we derive the electron temperature from an iterative procedure, using the equation from Aller (1984):

$t=\frac{1.432}{\log [(\lambda 4959+\lambda 5007) / \lambda 4363]-\log C_{T}}$,

where $t=10^{-4} T_{\mathrm{e}}(\mathrm{O}$ III $)$,

$C_{T}=\left(8.44-1.09 t+0.5 t^{2}-0.08 t^{3}\right) \frac{1+0.0004 x}{1+0.044 x}$,

and $x=10^{-4} N_{\mathrm{e}} t^{-0.5}$. The density $N_{\mathrm{e}}(\mathrm{S}$ II $)$ is derived by interpolation of the $[\mathrm{S}$ II $] \lambda 6717 / \lambda 6731$ line ratio vs. $N_{\mathrm{e}}(\mathrm{S}$ II) relation) with the use of the collisional strengths from Ramsbottom et al. (1996). The derived $N_{\mathrm{e}}$ is always smaller than $10^{3} \mathrm{~cm}^{-3}$, so that the term containing $x$ is never important.

In Fig. 2 we show the differences between the ionic abundances calculated with new and old atomic data as a function of the electron temperature. Each curve is labeled with the emission line used for the ionic abundance determination. We use [N II] $\lambda 6548,6584$ for the determination of the nitrogen abundance, [O II] $\lambda 3727$ (or [O II] $\lambda 7320,7331$ when [O II] $\lambda 3727$ is not available) and [O III] $\lambda 4959,5007$ for the oxygen abundance, [Ne III] $\lambda 3868$ for the neon abundance, [S III] $\lambda 6312$ and [S II] $\lambda 6717,6731$ for the sulfur abundance when detected, [Cl III] $\lambda 5517,5531$ for the chlorine abundance, [Ar III] $\lambda 7135$ (and [Ar IV] $\lambda 4740$ if seen) for the argon abundance, and [Fe III] $\lambda 4658$ for the iron abundance. In general, the new atomic data change the ionic abundances by not more than 0.06 dex. The only exception is $\mathrm{Ar}^{3+}$ where the new determinations are more than 0.1 dex lower than the old ones. Following Stasińska (2005) we do not attempt to analyze the collisional strengths for $\mathrm{Fe}^{2+}$ and use the same ones as in Thuan et al. (1995). We obtain linear fits for the dependences shown in Fig. 2 and use these fits in the abundance determinations. The ionic abundances are then derived from the equations:

$$
\begin{aligned}
12+\log \mathrm{O}^{+} / \mathrm{H}^{+}= & \log \frac{\lambda 3727}{\mathrm{H} \beta}+5.961+\frac{1.676}{t}-0.40 \log t \\
& -0.034 t+\log (1+1.35 x)
\end{aligned}
$$

$$
\begin{aligned}
12+\log \mathrm{O}^{+} / \mathrm{H}^{+}= & \log \frac{\lambda 7320+\lambda 7330}{\mathrm{H} \beta}+6.901 \\
& +\frac{2.487}{t}-0.483 \log t \\
& -0.013 t+\log (1-3.48 x),
\end{aligned}
$$

$$
\begin{aligned}
12+\log \mathrm{O}^{2+} / \mathrm{H}^{+}= & \log \frac{\lambda 4959+\lambda 5007}{\mathrm{H} \beta}+6.200+\frac{1.251}{t} \\
& -0.55 \log t-0.014 t
\end{aligned}
$$

$$
\begin{aligned}
12+\log \mathrm{N}^{+} / \mathrm{H}^{+}= & \log \frac{\lambda 6548+\lambda 6584}{\mathrm{H} \beta}+6.234 \\
& +\frac{0.950}{t}-0.42 \log t \\
& -0.027 t+\log (1+0.116 x)
\end{aligned}
$$

$$
\begin{aligned}
12+\log \mathrm{Ne}^{2+} / \mathrm{H}^{+}= & \log \frac{\lambda 3869}{\mathrm{H} \beta}+6.444+\frac{1.606}{t} \\
& -0.42 \log t-0.009 t
\end{aligned}
$$

$$
\begin{aligned}
12+\log \mathrm{S}^{+} / \mathrm{H}^{+}= & \log \frac{\lambda 6717+\lambda 6731}{\mathrm{H} \beta}+5.439 \\
& +\frac{0.929}{t}-0.28 \log t \\
& -0.018 t+\log (1+1.39 x),
\end{aligned}
$$

$$
\begin{aligned}
12+\log \mathrm{S}^{2+} / \mathrm{H}^{+}= & \log \frac{\lambda 6312}{\mathrm{H} \beta}+6.690+\frac{1.678}{t} \\
& -0.47 \log t-0.010 t
\end{aligned}
$$

$$
12+\log \mathrm{Cl}^{2+} / \mathrm{H}^{+}=\log \frac{\lambda 5517+\lambda 5537}{\mathrm{H} \beta}+5.700+\frac{1.088}{t}
$$$$
-0.483 \log t-0.002 t \text {, }
$$

$$
\begin{aligned}
12+\log \mathrm{Ar}^{2+} / \mathrm{H}^{+}= & \log \frac{\lambda 7135}{\mathrm{H} \beta}+6.174 \\
& +\frac{0.799}{t}-0.48 \log t \\
& -0.013 t+\log (1+0.22 x),
\end{aligned}
$$



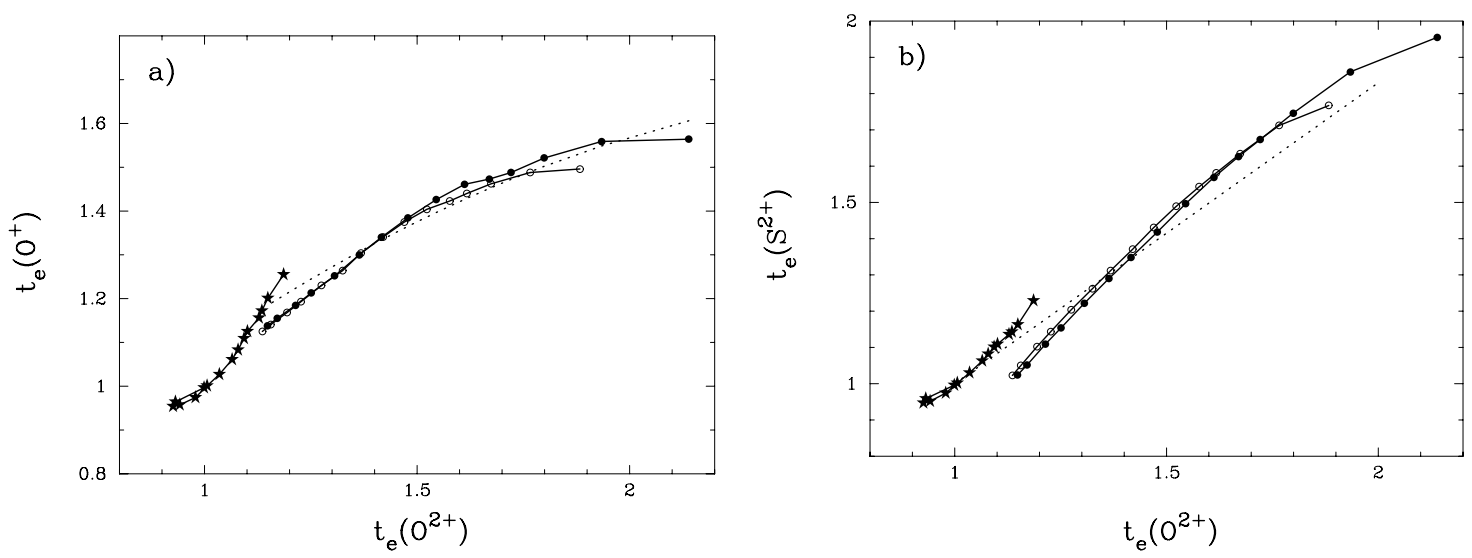

Fig. 3. Relations between the electron temperatures a) $t_{\mathrm{e}}(\mathrm{O}$ II $)$ and $t_{\mathrm{e}}(\mathrm{O}$ III $)\left(t_{\mathrm{e}}=10^{-4} T_{\mathrm{e}}\right)$ and b) $t_{\mathrm{e}}(\mathrm{S}$ III $)$ and $t_{\mathrm{e}}(\mathrm{O}$ III $)$ obtained from the sequences of photoionization models for H II galaxies (Stasińska \& Izotov 2003) using stellar atmosphere models from Smith et al. (2002). Filled circles are for the models with $Z=0.02 Z_{\odot}$, open circles are for the models with $Z=0.05 Z_{\odot}$, and stars are for the models with $Z=$ $0.2 Z_{\odot}$. The dotted lines are the approximation of the models of Stasińska (1990) in a) and the approximation of the models of Garnett (1992) in b).

$$
\begin{aligned}
12+\log \mathrm{Ar}^{3+} / \mathrm{H}^{+}= & \log \frac{\lambda 4740}{\mathrm{H} \beta}+6.308+\frac{1.280}{t} \\
& -0.48 \log t-0.047 t, \\
12+\log \mathrm{Fe}^{2+} / \mathrm{H}^{+}= & \log \frac{\lambda 4658}{\mathrm{H} \beta}+6.498+\frac{1.298}{t} \\
& -0.48 \log t .
\end{aligned}
$$

Since different ions reside in different parts of the $\mathrm{H}$ II region, the electron temperatures in Eqs. (3)-(13) are not the same for the various ions. Only $T_{\mathrm{e}}(\mathrm{O}$ III $)$ is derived directly from observations for all galaxies. Otherwise, we use the relations between $T_{\mathrm{e}}(\mathrm{O}$ III $)$ and the temperatures characteristic of other ions in sequences of photoionization models that best fit the observed line emission trends in $\mathrm{H}$ II galaxies. These sequences are defined as in Stasińska \& Izotov (2003), but have been recomputed with the new atomic data, and with an input radiation field computed with Starburst 99 (Leitherer et al. 1999) using the stellar model atmospheres described in Smith et al. (2002). We thus obtain the relations between the electron temperatures $T_{\mathrm{e}}(\mathrm{O}$ III $)$ and $T_{\mathrm{e}}(\mathrm{O}$ II $)$ which are shown by solid lines in Fig. $3 \mathrm{a}$. Adopting the nomenclature of Stasińska \& Izotov (2003), "low $Z$ " models are represented by filled circles, "intermediate $Z$ " models by open circles and "high $Z$ " models by stars. These $Z$ correspond to oxygen abundances $12+\log \mathrm{O} / \mathrm{H}=7.2,7.6$ and 8.2, respectively. For comparison, the dotted line is the fit of Izotov et al. $(1994,1997)$ to the earlier models of Stasińska (1990). The latter approximation, used in all our previous studies, is not very different from the new relations shown by solid lines in Fig. 3. We approximate the relations from the new models by the expressions

$$
\begin{aligned}
t(\mathrm{O} \text { II }) & =-0.577+t \times(2.065-0.498 t), \text { low } Z, \\
& =-0.744+t \times(2.338-0.610 t), \text { intermed. } Z, \\
& =2.967+t \times(-4.797+2.827 t), \text { high } Z,
\end{aligned}
$$

where $t(\mathrm{O}$ II $)=10^{-4} T\left(\mathrm{O}\right.$ II) and $t=10^{-4} T(\mathrm{O}$ III).

In the $\mathrm{H}$ II regions where both [O $\mathrm{OI}] \lambda 3727$ and [O II] 27320,7331 are detected, the electron temperature $T_{\mathrm{e}}(\mathrm{O}$ II) can be derived directly from the $\lambda 3727 /(\lambda 7320+\lambda 7331)$ ratio. In Fig. 4a we compare the relations between $T_{\mathrm{e}}(\mathrm{O}$ II $)$ and $T_{\mathrm{e}}(\mathrm{O}$ III $)$ obtained from the observations with those from model predictions. Large filled circles correspond to galaxies from the HeBCD sample, while dots correspond to SDSS galaxies. Despite a large scatter of the data points caused mainly by large flux errors of the weak [O II] $\lambda 7320,7331$ emission lines, the relation between $T_{\mathrm{e}}(\mathrm{O}$ II $)$ and $T_{\mathrm{e}}(\mathrm{O}$ III $)$ derived from the observations follows generally the one obtained from the models. Therefore, in the following we adopt the model relation to derive $T_{\mathrm{e}}(\mathrm{O}$ II $)$. Note that the scatter in Fig. 4 implies that, for the low redshift objects of the SDSS sample for which [O II] $\lambda 3727$ is outside the observed wavelength range, the $\mathrm{O}^{+}$abundance is not very accurate since it is obtained from [O II] 17320,7331 . This concerns about $30 \%$ of the sample.

Similar relations between $T_{\mathrm{e}}(\mathrm{S}$ III $)$ and $T_{\mathrm{e}}(\mathrm{O}$ III $)$ can be obtained from the same photoionization models:

$$
\begin{aligned}
t(\mathrm{~S} \text { III }) & =-1.085+t \times(2.320-0.420 t), \text { low } Z, \\
& =-1.276+t \times(2.645-0.546 t), \text { intermed. } Z, \\
& =1.653+t \times(-2.261+1.605 t), \text { high } Z,
\end{aligned}
$$

where $t(\mathrm{~S}$ III $)=10^{-4} T_{\mathrm{e}}(\mathrm{S}$ III $)$ and $t=10^{-4} T_{\mathrm{e}}(\mathrm{O}$ III $)$. They are shown in Fig. 3b. The large wavelength coverage of the SDSS spectra allows us to detect at the same time the auroral [S III] $\lambda 6312$ and nebular [S III] $\lambda 9069$ emission lines in low redshift galaxies and to derive the electron temperature $T_{\mathrm{e}}$ (S III). In Fig. 4b we show the dependence of $T_{\mathrm{e}}(\mathrm{S}$ III) on $T_{\mathrm{e}}(\mathrm{O}$ III) for those galaxies. Again, the theoretical relation is in satisfactory agreement with the observations, if we take into account the large scatter in the data, mostly due to measurement errors of the weak [S III] $\lambda 6312$ line.

We thus adopt $T_{\mathrm{e}}(\mathrm{O}$ II) from Eq. (14) for the calculation of $\mathrm{N}^{+}, \mathrm{O}^{+}, \mathrm{S}^{+}$and $\mathrm{Fe}^{2+}$ abundances, $T_{\mathrm{e}}(\mathrm{S}$ III) from Eq. (15) for the calculation of $\mathrm{S}^{2+}, \mathrm{Cl}^{2+}$ and $\mathrm{Ar}^{2+}$ abundances and $T_{\mathrm{e}}\left(\mathrm{O}\right.$ III) from Eq. (1) for the calculation of $\mathrm{O}^{2+}$ and $\mathrm{Ne}^{2+}$ abundances. We adopt the "low $Z$ " fits for $\mathrm{H}$ II regions with $12+\log \mathrm{O} / \mathrm{H} \leq 7.2$, the "high $Z$ " fits for the $\mathrm{H}$ II regions with 

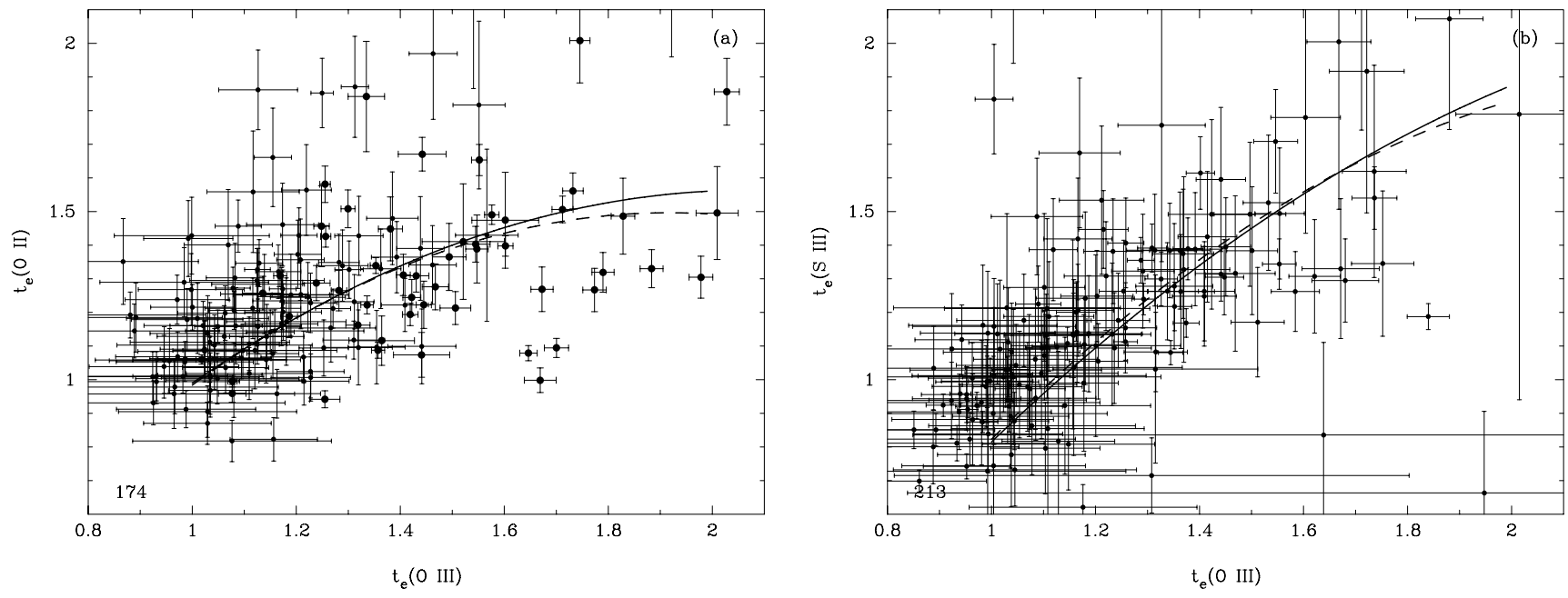

Fig. 4. a) Relation between the electron temperatures $t_{\mathrm{e}}(\mathrm{O}$ II $)$ and $t_{\mathrm{e}}(\mathrm{O}$ III) for the SDSS sample (small circles) and HeBCD galaxies (large circles). b) Relation between the electron temperatures $t_{\mathrm{e}}(\mathrm{S}$ III $)$ and $t_{\mathrm{e}}(\mathrm{O}$ III $)$ for the SDSS sample. The solid and dashed lines in a) and b) are respectively the predictions for $\mathrm{H}$ II region models (Eqs. (14)-(15)) with low- and intermediate metallicities.

$12+\log \mathrm{O} / \mathrm{H} \geq 8.2$ (Eqs. (14) and (15)), and linearly interpolate $T_{\mathrm{e}}(\mathrm{O}$ II $)$ and $T_{\mathrm{e}}(\mathrm{S}$ III $)$ between "low $Z$ " and "intermediate $Z$ " cases for $\mathrm{H}$ II regions with $12+\log \mathrm{O} / \mathrm{H}$ between 7.2 and 7.6, and between "intermediate $Z$ " and "high $Z$ " cases for $\mathrm{H}$ II regions with $12+\log \mathrm{O} / \mathrm{H}$ between 7.6 and 8.2. Since the population of the $\mathrm{Ar}^{3+}$ ion is generally smaller than that of the $\mathrm{Ar}^{2+}$ ion and the [Ar IV] lines weaker than the [Ar III] lines, we decided to derive the Ar abundance only from the abundance of the $\mathrm{Ar}^{2+}$ ion.

\subsection{Ionization correction factors}

The total element abundance can be derived from the abundances of ions seen in the optical spectra using ionization correction factors $(I C F \mathrm{~s})$. For this, the ionization structure of the $\mathrm{H}$ II region has to be derived from photoionization models. Fortunately, the most abundant ions of oxygen, $\mathrm{O}^{+}$and $\mathrm{O}^{2+}$, are seen in the optical spectra of the $\mathrm{H}$ II regions. This fact allows one to immediately derive the oxygen abundance as

$\frac{\mathrm{O}}{\mathrm{H}}=\frac{\mathrm{O}^{+}}{\mathrm{H}^{+}}+\frac{\mathrm{O}^{2+}}{\mathrm{H}^{+}}$.

The small fraction of the unseen $\mathrm{O}^{3+}$ ion in the high-excitation $\mathrm{H}$ II regions can be added to the oxygen abundance if the He II $\lambda 4686$ emission line is detected in their spectra. Our sequences of photoionization models show that $\mathrm{O}^{3+} / \mathrm{O}$ is $\gtrsim 1 \%$ only in the highest-excitation $\mathrm{H}$ II regions with $\mathrm{O}^{+} /\left(\mathrm{O}^{+}+\mathrm{O}^{2+}\right)$ $\lesssim 0.1$. In those $\mathrm{H}$ II regions, $\mathrm{O}^{3+} / \mathrm{H}^{+}$can be approximated by

$\frac{\mathrm{O}^{3+}}{\mathrm{H}^{+}}=0.5 \times \frac{\mathrm{He}^{2+}}{\mathrm{He}^{+}+\mathrm{He}^{2+}}\left(\frac{\mathrm{O}^{+}}{\mathrm{H}^{+}}+\frac{\mathrm{O}^{2+}}{\mathrm{H}^{+}}\right)$.

This results in a minimal correction to the oxygen abundance derived just from $\mathrm{O}^{+}$and $\mathrm{O}^{2+}$. In the lower-excitation $\mathrm{H}$ II regions with $\mathrm{O}^{+} /\left(\mathrm{O}^{+}+\mathrm{O}^{2+}\right)>0.1$, we set $\mathrm{O}^{3+} / \mathrm{H}^{+}$to zero.

The ionization structure of an $\mathrm{H}$ II region is determined by the balance between the processes of photoionization and recombination. The rates for some of these processes are poorly

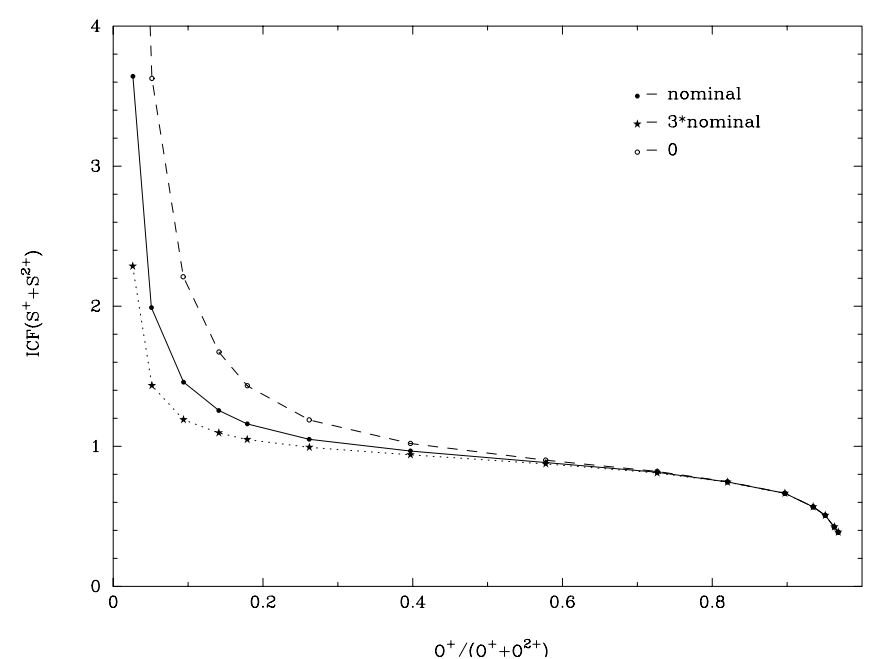

Fig. 5. Ionization correction factor $I C F\left(\mathrm{~S}^{+}+\mathrm{S}^{2+}\right)$ vs. the fraction $\mathrm{O}^{+} /\left(\mathrm{O}^{+}+\mathrm{O}^{2+}\right)$ for the "low $Z$ " models with different rates of dielectronic recombination for sulfur ions. Nominal dielectronic recombination for sulfur ions is the same as that for oxygen ions.

known. One of the major problems is the uncertain rate of the dielectronic recombination for sulfur, chlorine and argon ions. The comparison of the abundances of these elements calculated with different assumed dielectronic recombination rates allows us to put some constraints on these rates. Figure 5 shows the dependence of $I C F\left(\mathrm{~S}^{+}+\mathrm{S}^{2+}\right)$ with $\mathrm{O}^{+} /\left(\mathrm{O}^{+}+\mathrm{O}^{2+}\right)$ for the "low $Z$ " models. The solid curve corresponds to what we call the "nominal" dielectronic recombination rates (equal to the dielectronic recombination rates for second-row elements in the same isoelectronic sequence). We adopt as the nominal dielectronic recombination rates for $\mathrm{S}$ ions the same as for $\mathrm{O}$ ions. The dotted line corresponds to dielectronic recombination rates enhanced by a factor 3 , and the dashed one is obtained with a total suppression of dielectronic recombination. It is seen from this figure that $I C F\left(\mathrm{~S}^{+}+\mathrm{S}^{2+}\right)$ decreases when the dielectronic recombination rate increases and the effect is more important 

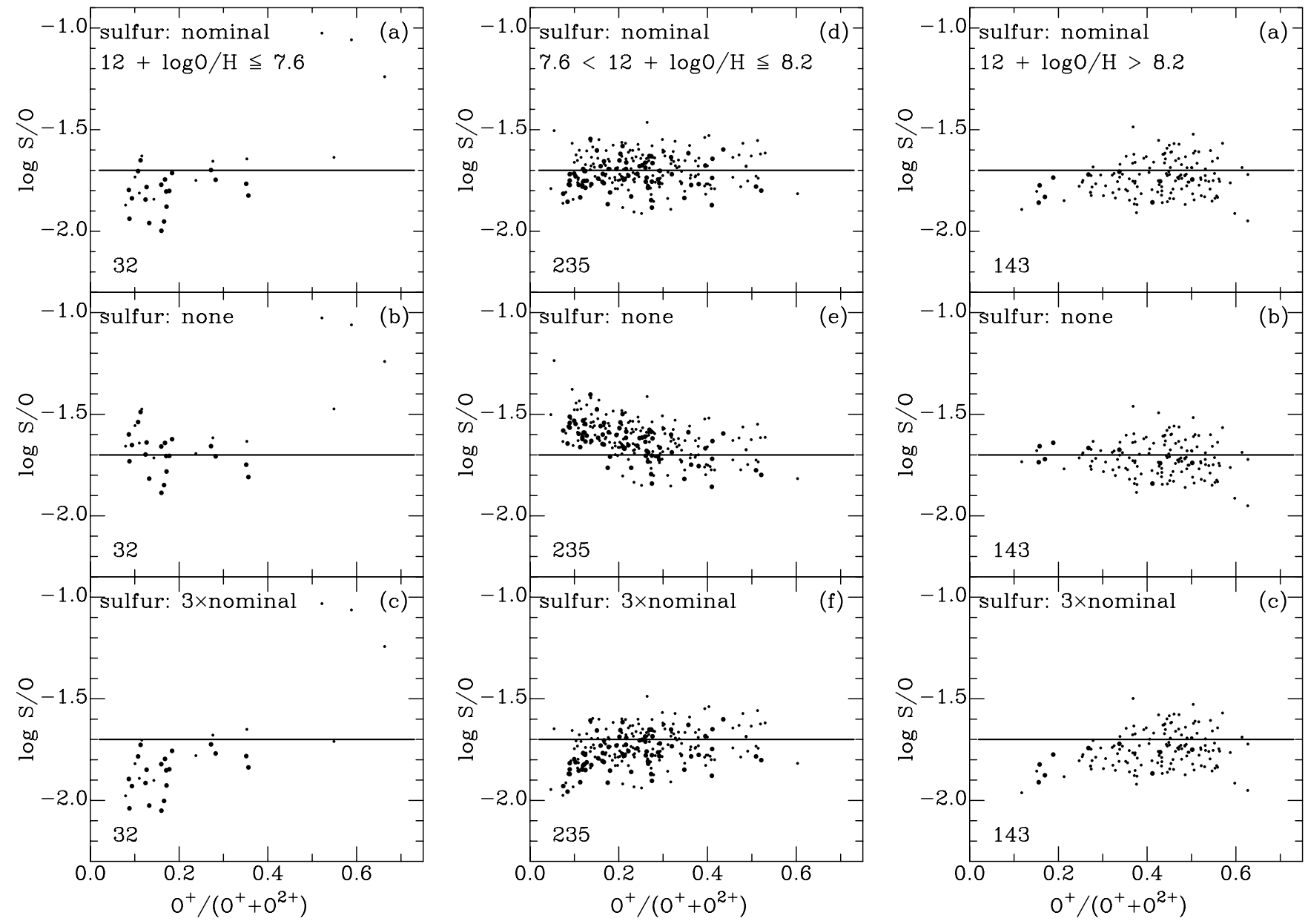

Fig. 6. Left: sulfur-to-oxygen abundance ratio vs. the fraction $\mathrm{O}^{+} /\left(\mathrm{O}^{+}+\mathrm{O}^{2+}\right)$ in the $\mathrm{H}$ II regions with $12+\log \mathrm{O} / \mathrm{H} \leq 7.6$ for different rates of dielectronic recombination for sulfur. The nominal dielectronic recombination for $\mathrm{S}$ ions is the same as that for $\mathrm{O}$ ions. Large filled circles are data for the HeBCD sample, dots are for the SDSS sample. Solid horizontal line shows the mean S/O abundance ratio from the data with the nominal rate of dielectronic recombination for sulfur ions. Middle: the same as in the left panel but for $\mathrm{H}$ II regions with $7.6<12+\log \mathrm{O} / \mathrm{H} \leq$ 8.2. Right: the same as in the left panel but for $\mathrm{H}$ II regions with $12+\log \mathrm{O} / \mathrm{H}>8.2$. The total number of galaxies in each panel is shown in the lower left corner.

for high-excitation models. Figure 6 shows the values of S/O calculated with different dielectronic recombination rates as a function of the fraction $\mathrm{O}^{+} /\left(\mathrm{O}^{+}+\mathrm{O}^{2+}\right)$ for the galaxies in the SDSS and HeBCD combined sample. Since the effect of dielectronic recombination may depend on the metallicity in the $\mathrm{H}$ II region, via the electron temperature, we split the sample into three metallicity bins: the hottest $\mathrm{H}$ II regions with $12+$ $\log \mathrm{O} / \mathrm{H} \leq 7.6$ (left panel), the intermediate-temperature $\mathrm{H}$ II regions with $7.6<12+\log \mathrm{O} / \mathrm{H} \leq 8.2$ (middle panel) and the coolest $\mathrm{H}$ II regions with $12+\log \mathrm{O} / \mathrm{H}>8.2$ (right panel). It is expected that if the adopted rate of dielectronic recombination for sulfur ions is correct then there should be no dependence of the derived $\mathrm{S} / \mathrm{O}$ with $\mathrm{O}^{+} /\left(\mathrm{O}^{+}+\mathrm{O}^{2+}\right)$. Inspection of Fig. 6 shows that trends in the $\mathrm{S} / \mathrm{O}$ abundance ratio are minimal if a zero rate of dielectronic recombination for sulfur ions is adopted for the low- and high-metallicity bins and the nominal rate is adopted for the intermediate-metallicity bin. Thus, we adopt the $I C F \mathrm{~s}\left(\mathrm{~S}^{+}+\mathrm{S}^{2+}\right)$ calculated with a zero rate for dielectronic recombination for the low- and high-metallicity bins and with a nominal rate for the intermediate-metallicity bin.
In a similar way, we consider the effect of dielectronic recombination on the ICF s for argon. We adopt as the nominal dielectronic recombination rate the same values as for ions of neon. Figure 7 shows the dependence of the Ar/O abundance ratio with $\mathrm{O}^{+} /\left(\mathrm{O}^{+}+\mathrm{O}^{2+}\right)$. Similarly to $\mathrm{S} / \mathrm{O}$, the value of $\mathrm{Ar} / \mathrm{O}$ should not depend on $\mathrm{O}^{+} /\left(\mathrm{O}^{+}+\mathrm{O}^{2+}\right)$. A minimal trend in the $\mathrm{Ar} / \mathrm{O}$ abundance ratio is seen for the $\mathrm{H}$ II regions in the whole range of metallicities if the nominal rate is adopted. A similar consideration for chlorine (Fig. 8) shows that minimal trends are present for the $\mathrm{H}$ II regions with $12+\log \mathrm{O} / \mathrm{H}>7.6$ if a zero rate is adopted. As for lower-metallicity $\mathrm{H}$ II regions, the statistics are too poor to make definite conclusions. We adopt above as the nominal dielectronic recombination rates for $\mathrm{Cl}$ ions the same as for $\mathrm{O}$ ions. In the following, we adopt the $I C F \mathrm{~s}$ derived from the models with a nominal rate for dielectronic recombination for argon ions and a zero rate for chlorine ions.

The adopted $I C F$ s to obtain the elemental abundances from the ionic ones are shown in Fig. 9, as a function of $\mathrm{O}^{+} /\left(\mathrm{O}^{+}+\mathrm{O}^{2+}\right)$ or $\mathrm{O}^{2+} /\left(\mathrm{O}^{+}+\mathrm{O}^{2+}\right)$. As in Fig. 3, filled circles, 

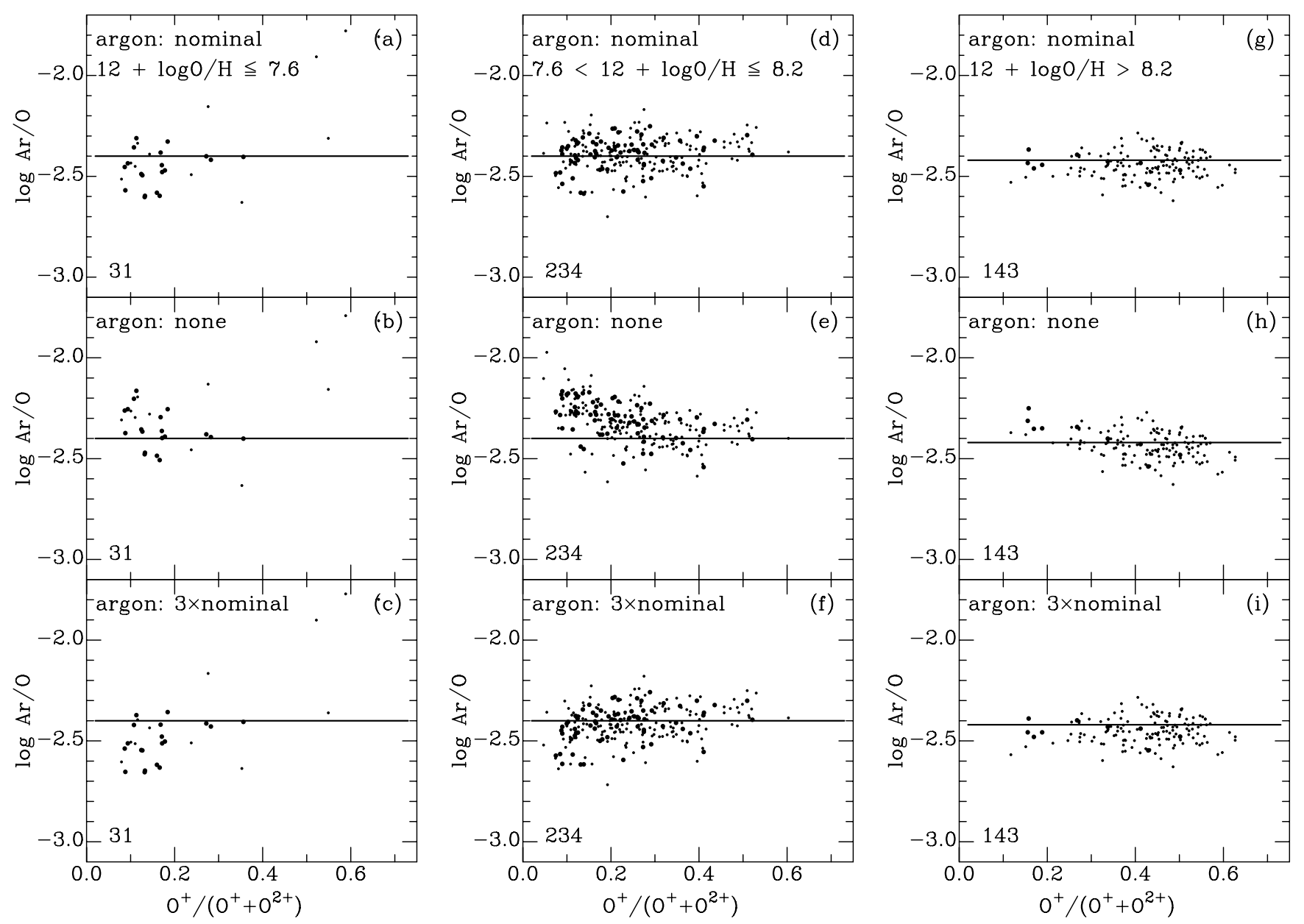

Fig. 7. The same as in Fig. 6 but for argon. Nominal dielectronic recombination for Ar ions is the same as that for Ne ions.

open circles and stars correspond to the $\mathrm{H}$ II region models with $Z=0.02 Z_{\odot}, 0.05 Z_{\odot}$ and $0.2 Z_{\odot}$, respectively. Dotted lines are approximations of $I C F \mathrm{~s}$ from the Stasińska (1990) models.

It is seen from Fig. 9 that there is very little dependence of $I C F\left(\mathrm{~N}^{+}\right), I C F\left(\mathrm{Cl}^{2+}\right), I C F\left(\mathrm{Ar}^{2+}\right)$ and $I C F\left(\mathrm{Fe}^{2+}\right)$ on metallicity over the whole range of oxygen abundance considered here. On the other hand, $I C F\left(\mathrm{Ne}^{2+}\right)$ and $I C F\left(\mathrm{~S}^{+}+\mathrm{S}^{2+}\right)$ are different for the $Z=0.02$ and $0.05 Z_{\odot}$ model sequences as compared to the $Z=0.2 Z_{\odot}$ sequence. This is due to the fact that the $Z=$ 0.02 and $0.05 Z_{\odot}$ model sequences include an $\mathrm{X}$-ray ionizing source in addition to the ionizing star population. This was required in order to account for the large proportion of galaxies with nebular He II 24686 . If an X-ray source is present, it creates in the $\mathrm{H}$ II region an extended warm zone where hydrogen is partly recombined, and where $\mathrm{O}^{2+}$ ions are decoupled from $\mathrm{Ne}^{2+}$ ions due to the efficiency of the $\mathrm{O}^{2+}+\mathrm{H}^{0}$ charge transfer. At present, we have no real clue on the nature and strength of these X-ray sources, so the $I C F$ for neon in low excitation $\mathrm{H}$ II regions with $12+\log \mathrm{O} / \mathrm{H} \leq 8.2$ is uncertain. Fortunately, such cases do not represent a large fraction of our sample, as can be seen in Figs. 6-8.

The new $I C F \mathrm{~s}$ for nitrogen and iron are undistinguishable from the ones obtained from the Stasińska (1990) models, while for other elements there are significant differences. We fit the new $I C F$ s by the expressions

$$
\begin{aligned}
\operatorname{ICF}\left(\mathrm{N}^{+}\right) & =-0.825 v+0.718+0.853 / v, \text { low } Z, \\
& =-0.809 v+0.712+0.852 / v, \text { intermed. } Z, \\
& =-1.476 v+1.752+0.688 / v, \text { high } Z,
\end{aligned}
$$

$$
\begin{aligned}
I C F\left(\mathrm{Ne}^{2+}\right) & =-0.385 w+1.365+0.022 / w, \text { low } Z, \\
& =-0.405 w+1.382+0.021 / w, \text { intermed. } Z, \\
& =-0.591 w+0.927+0.546 / w, \text { high } Z,
\end{aligned}
$$

$$
\begin{aligned}
I C F\left(\mathrm{~S}^{+}+\mathrm{S}^{2+}\right) & =0.121 v+0.511+0.161 / v, \text { low } Z \\
& =0.155 v+0.849+0.062 / v, \text { inter. } Z, \\
& =0.178 v+0.610+0.153 / v, \text { high } Z
\end{aligned}
$$

$$
\begin{aligned}
I C F\left(\mathrm{Cl}^{2+}\right) & =0.756 v+0.648+0.128 / v, \text { low } Z, \\
& =0.814 v+0.620+0.131 / v, \text { intermed. } Z, \\
& =1.186 v+0.357+0.121 / v, \text { high } Z,
\end{aligned}
$$

$$
\begin{aligned}
\operatorname{ICF}\left(\mathrm{Ar}^{2+}\right) & =0.278 v+0.836+0.051 / v, \text { low } Z, \\
& =0.285 v+0.833+0.051 / v, \text { intermed. } Z, \\
& =0.517 v+0.763+0.042 / v, \text { high } Z,
\end{aligned}
$$



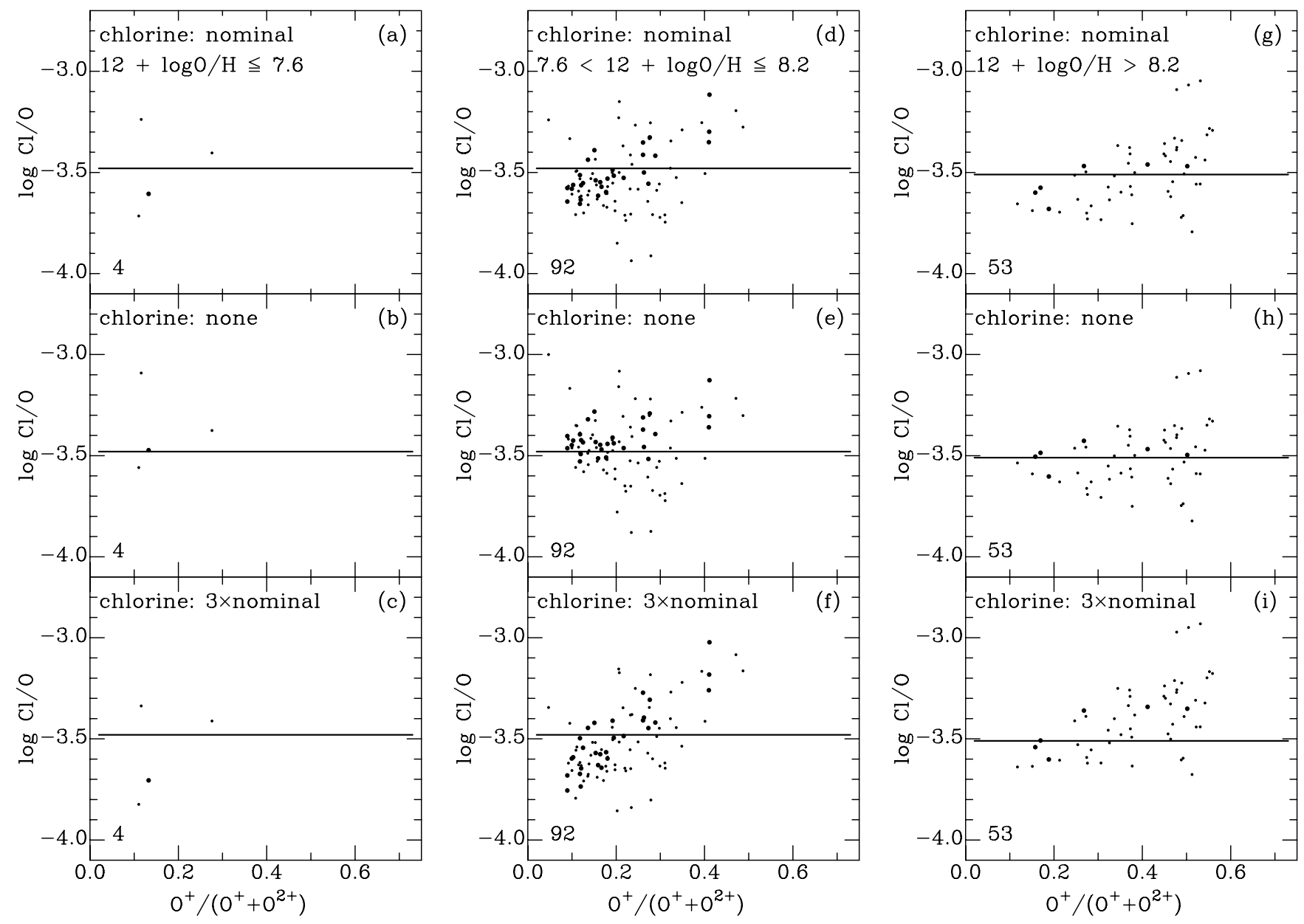

Fig. 8. The same as in Fig. 6 but for chlorine. Nominal dielectronic recombination for $\mathrm{Cl}$ ions is the same as that for $\mathrm{O}$ ions.

$$
\begin{aligned}
I C F\left(\mathrm{Ar}^{2+}+\mathrm{Ar}^{3+}\right) & =0.158 v+0.958+0.004 / v, \text { low } Z, \\
& =0.104 v+0.980+0.001 / v, \text { int. } Z, \\
& =0.238 v+0.931+0.004 / v, \text { high } Z,
\end{aligned}
$$

$$
\begin{aligned}
I C F\left(\mathrm{Fe}^{2+}\right) & =0.036 v-0.146+1.386 / v, \text { low } Z, \\
& =0.301 v-0.259+1.367 / v, \text { intermed. } Z, \\
& =-1.377 v+1.606+1.045 / v, \text { high } Z,
\end{aligned}
$$

where $v=\mathrm{O}^{+} /\left(\mathrm{O}^{+}+\mathrm{O}^{2+}\right)$ and $w=\mathrm{O}^{2+} /\left(\mathrm{O}^{+}+\mathrm{O}^{2+}\right)$. The fits given by Eqs. (18), (19) and (24) are applicable over the whole range of $v, w=0-1$, while the remaining fits are applicable only for $v \lesssim 0.8$. We note however that only a few $\mathrm{H}$ II regions in our sample have $v>0.8$. We adopt the "low $Z$ " ICF s for H II regions with $12+\log \mathrm{O} / \mathrm{H} \leq 7.2$, "high $Z$ " ICFs for $\mathrm{H}$ II regions with $12+\log \mathrm{O} / \mathrm{H} \geq 8.2$ and linearly interpolated $I C F$ s between "low $Z$ " and "intermediate $Z$ " cases for H II regions with $12+$ $\log \mathrm{O} / \mathrm{H}$ between 7.2 and 7.6, and between "intermediate $Z$ " and "high $Z$ " cases for $\mathrm{H}$ II regions with $12+\log \mathrm{O} / \mathrm{H}$ between 7.6 and 8.2. Although ICFs for some elements (e.g., for nitrogen) appear very similar in all metallicity bins (Fig. 9), we show in Eqs. (18)-(24) fits obtained separately for each bin.

In such a way, we can derive all elemental abundances using only analytical formulae. This is useful for the derivation of the formal uncertainties in the derived abundances, since they can be obtained by expanding the formulae in Taylor series, and do not require the use of Monte-Carlo methods.

\section{Discussion}

\subsection{SDSS DR3 galaxies versus galaxies from the HeBCD sample}

Electron temperatures $t_{\mathrm{e}}(\mathrm{O}$ III), element abundances and abundance ratios in the $\mathrm{H}$ II regions from the SDSS DR3 sample calculated as explained above are shown in Table 2. Some objects from the SDSS sample are also present in the HeBCD sample. For those objects we compare in Table 3 oxygen abundances derived from different observations. The SDSS spectra of these galaxies are shown in Fig. 10. Since the redshifts of these galaxies are small, the [O II] $\lambda 3727$ is not in the observed wavelength range of the SDSS spectra and therefore the $\mathrm{O}^{+}$ abundance was obtained from the [O II] 17320,7331 emission line fluxes. Exceptions are HS 0837+4717, HS 0924+3821, SBS $0948+532$ and SBS $1358+576$ with higher redshifts where the $\mathrm{O}^{+}$abundance was obtained from the [O II] $\lambda 3727$ emission line fluxes. The galaxies HS $0837+4717$, Mrk 1236, SBS 0917+527, UM 461 and UM 462 were observed twice in the course of SDSS and we show both oxygen abundances for each 

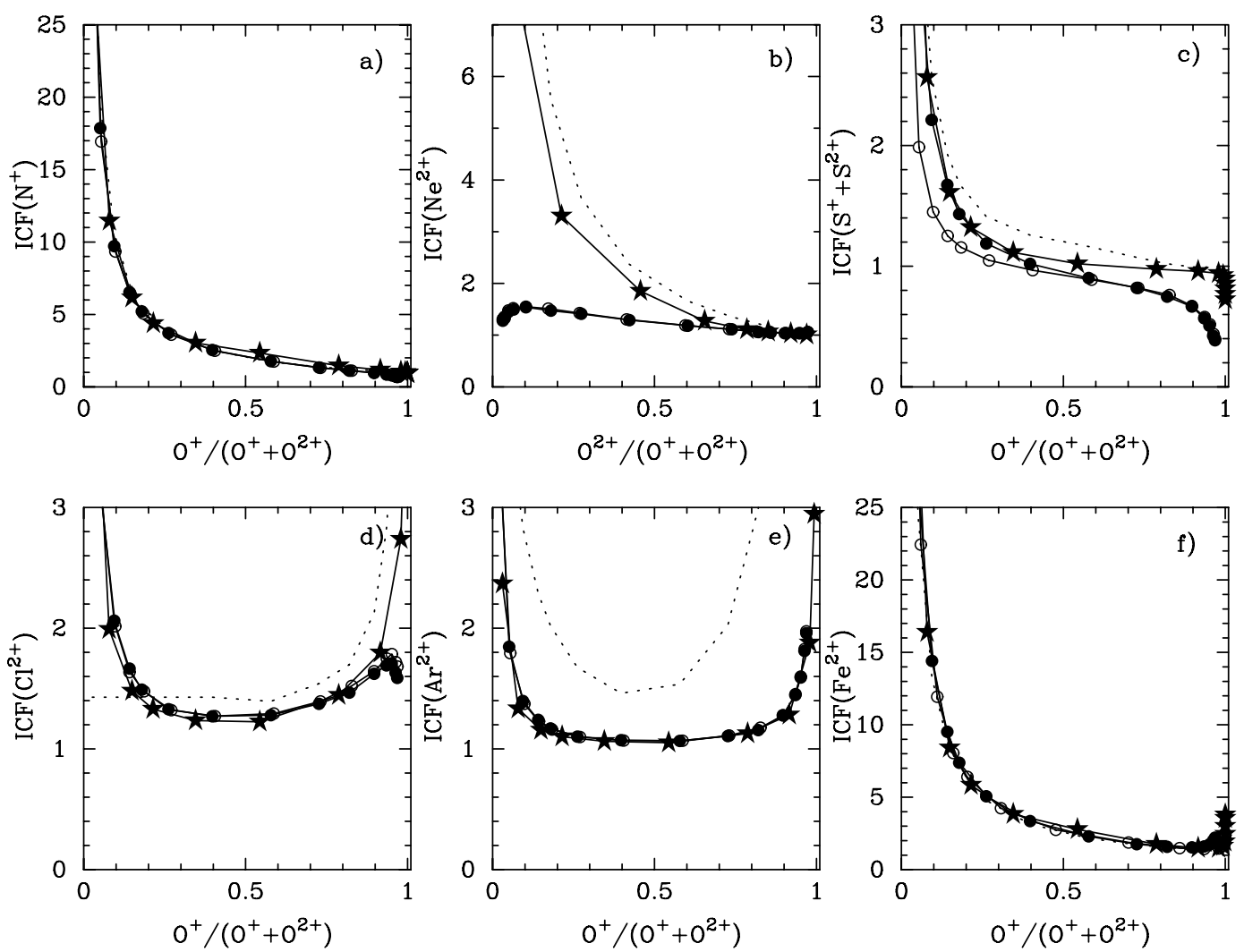

Fig. 9. Ionization correction factors for heavy elements. Filled circles are for the models with $Z=0.02 Z_{\odot}$, open circles are for the models with $Z=0.05 Z_{\odot}$, and stars are for the models with $Z=0.2 Z_{\odot}$. The dotted line is the approximation of the models of Stasińska (1990).

Table 2. Element abundances in the SDSS galaxies ${ }^{1}$

\begin{tabular}{lccccccc}
\hline \hline Spectrum number & $t_{\mathrm{e}}(\mathrm{O} \mathrm{III})$ & $12+\log \mathrm{O} / \mathrm{H}$ & $\log \mathrm{N} / \mathrm{O}$ & $\log \mathrm{Ne} / \mathrm{O}$ & \multicolumn{1}{c}{$\log \mathrm{S} / \mathrm{O}$} & \multicolumn{1}{c}{$\log \mathrm{Ar} / \mathrm{O}$} & $\log \mathrm{Fe} / \mathrm{O}$ \\
\hline $0266-51630-100$ & $1.5533 \pm 0.0327$ & $7.84 \pm 0.02$ & $-1.62 \pm 0.03$ & $-0.85 \pm 0.04$ & $-1.68 \pm 0.04$ & $-2.40 \pm 0.04$ & $-1.93 \pm 0.14$ \\
$0267-51608-384$ & $1.2055 \pm 0.0600$ & $7.99 \pm 0.05$ & $-1.30 \pm 0.07$ & $-0.55 \pm 0.10$ & $-1.71 \pm 0.11$ & $-2.43 \pm 0.07$ & $-1.69 \pm 0.21$ \\
$0270-51909-306$ & $0.9444 \pm 0.1699$ & $8.33 \pm 0.20$ & $-1.09 \pm 0.26$ & $-0.77 \pm 0.41$ & $-1.67 \pm 0.33$ & $\ldots$ & $\ldots$ \\
$0272-51941-365$ & $1.0133 \pm 0.1661$ & $8.38 \pm 0.18$ & $-1.40 \pm 0.23$ & $-0.65 \pm 0.36$ & $-1.60 \pm 0.29$ & $-2.50 \pm 0.22$ & $-2.32 \pm 0.80$ \\
$0275-51910-429$ & $1.5117 \pm 0.0513$ & $7.82 \pm 0.03$ & $-1.45 \pm 0.05$ & $-0.72 \pm 0.06$ & $-1.73 \pm 0.10$ & $-2.37 \pm 0.05$ & $\ldots$ \\
\hline
\end{tabular}

${ }^{1}$ Only the first five galaxies are shown. The total list of galaxies is available in electronic form at the CDS. The $\mathrm{Cl} / \mathrm{O}$ abundance ratios are not shown here for lack of space, but they are present in the electronic table.

of those galaxies. It is seen from Table 3 that $12+\log \mathrm{O} / \mathrm{H}$ derived from different observations are generally in good agreement. One exception is the galaxy UM 461. In one of two SDSS observations of this galaxy, the [O III] 24959,5007 emission lines are clipped resulting in a low oxygen abundance. The oxygen abundances for some galaxies from Table 3 are also in agreement with independent abundance determinations from the same SDSS spectra by Kniazev et al. (2003, 2004).

In Fig. 11 we show the computed N/O, Ne/O, S/O, Cl/O, $\mathrm{Ar} / \mathrm{O}, \mathrm{Fe} / \mathrm{O}$ abundance ratios vs. oxygen abundance $12+$ $\log \mathrm{O} / \mathrm{H}$ for the SDSS and HeBCD merged sample using old (left panels) and new (right panels) expressions to derive the abundances. In all panels, the HeBCD galaxies are represented by large filled circles, while the SDSS galaxies are shown by dots. In each panel, the solar abundance ratio (as compiled by Lodders 2003) is represented by a large open circle.
It is seen from Fig. 11 that SDSS galaxies are in general more metal-rich than the galaxies from the HeBCD sample. Only a few SDSS H II regions have an oxygen abundance $12+\log \mathrm{O} / \mathrm{H}$ smaller than 7.6. Two of them are actually the northwest and the southeast components of the well-known metal-deficient star-forming dwarf galaxy I Zw 18, which are also present in the HeBCD sample. Thus, star-forming galaxies with extremely low metallicity are very rare in the spectroscopic data base of the SDSS.

The distribution of the SDSS DR3 galaxies in the different panels of Fig. 11 confirms previous findings concerning HeBCD (Thuan et al. 1995; Izotov \& Thuan 1999) and SDSS Early Data Release galaxies (Izotov et al. 2004). The use of the new expressions to compute elemental abundances (Fig. 11, middle and right panels) result in very slight changes. $\mathrm{Ne} / \mathrm{O}$ abundance ratios are typically decreased by 0.1 dex. The largest 

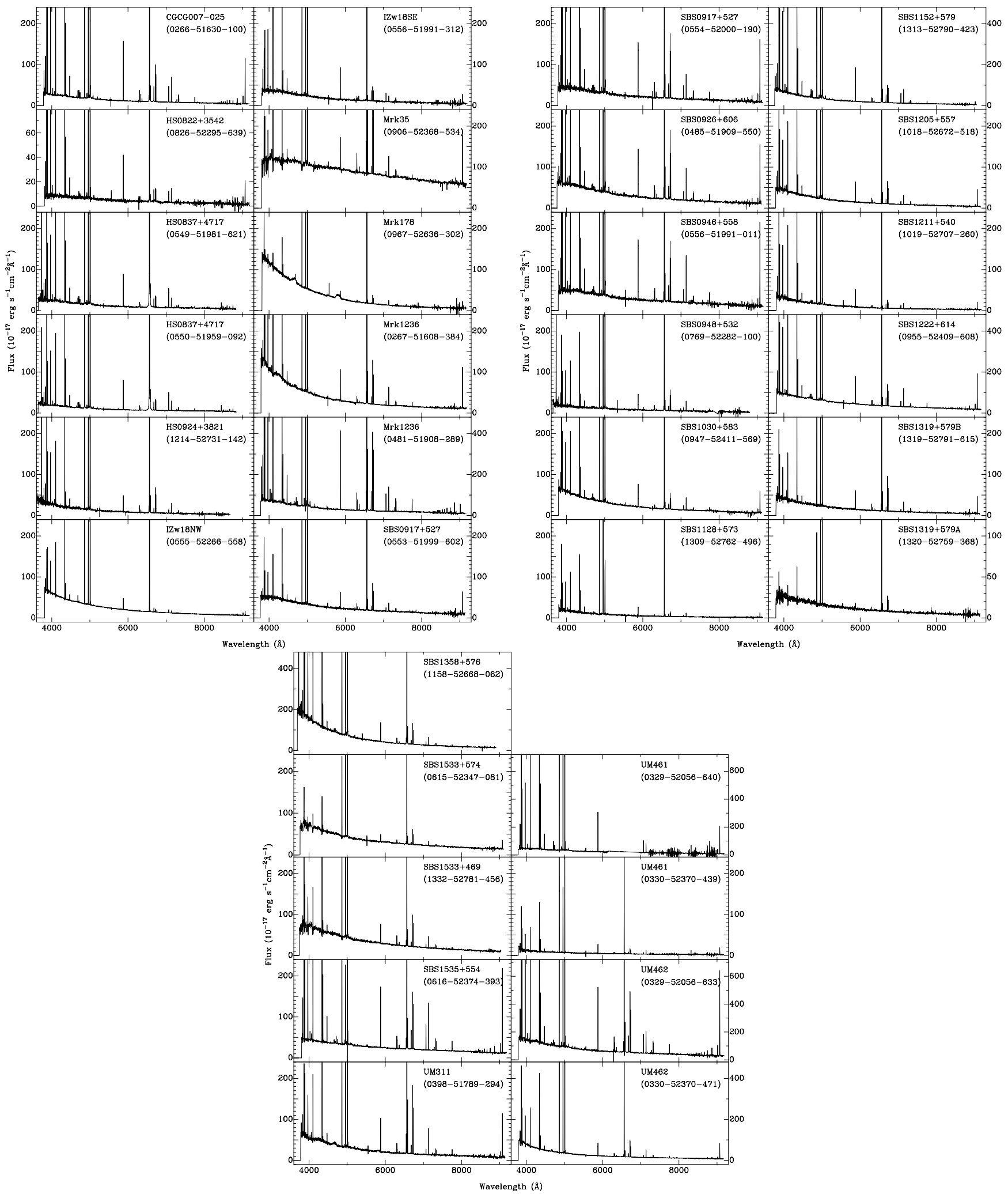

Fig. 10. SDSS spectra of the H II regions listed in Table 3. The names of the spectra in the SDSS database are shown in parentheses.

effect is for the $\mathrm{S} / \mathrm{O}$ and $\mathrm{Ar} / \mathrm{O}$ abundance ratios, which decrease typically by 0.15 dex.

The mean $\mathrm{Ne} / \mathrm{O}$ and $\mathrm{Cl} / \mathrm{O}$ abundance ratios for our galaxies are in good agreement with the solar abundance ratios from Lodders (2003) (open circles in Fig. 11). On the other hand, the
$\mathrm{S} / \mathrm{O}$ and $\mathrm{Ar} / \mathrm{O}$ abundance ratios in the $\mathrm{H}$ II regions are lower than the solar abundance ratios (by 0.15 dex and 0.25 dex, respectively). We cannot exclude the possibility that these differences are caused by uncertainties in the determination of $S$ and Ar abundances in the H II regions. Alternatively, they may 

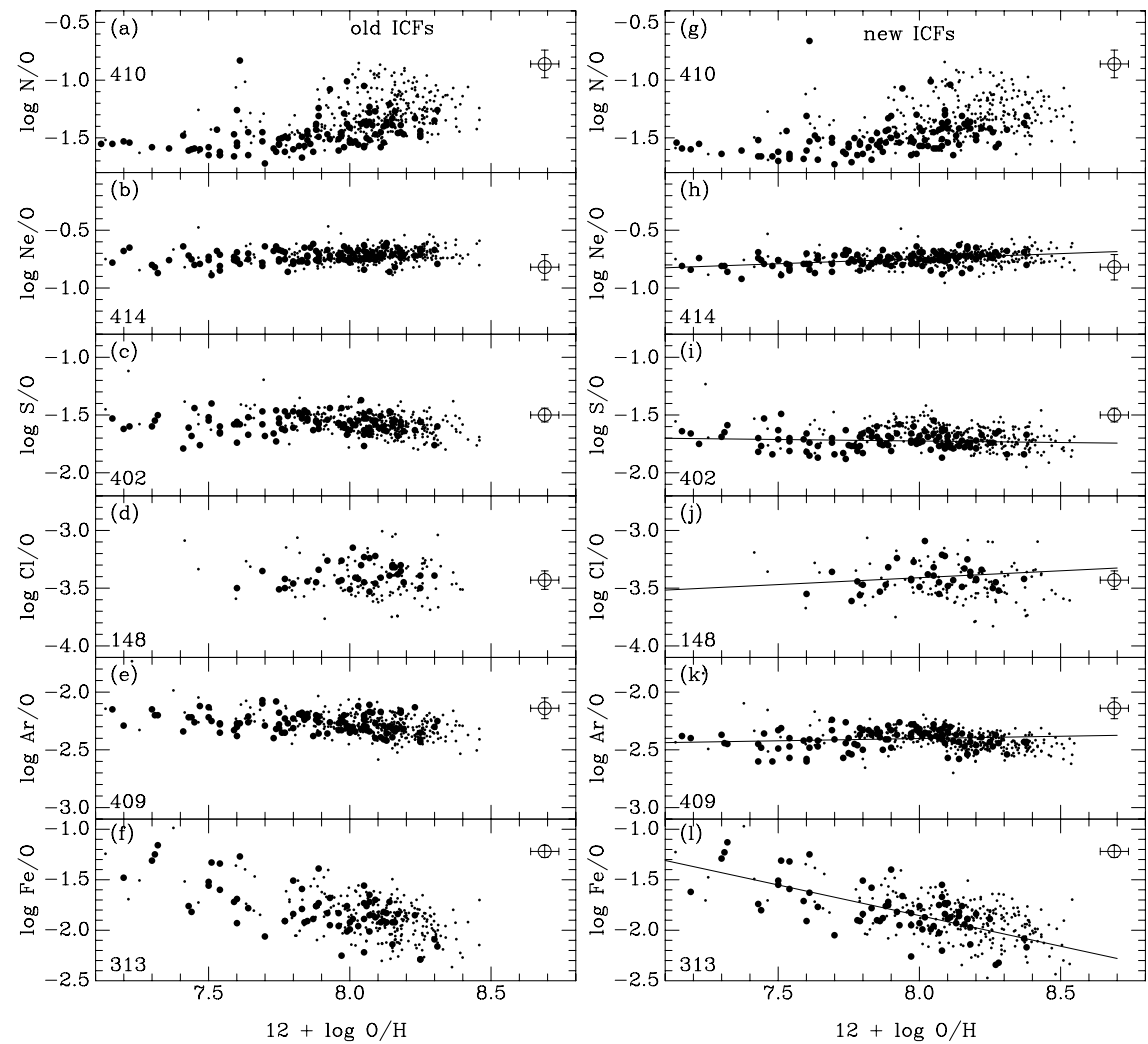

Fig. 11. $\log \mathrm{N} / \mathrm{O}$ a), g), $\log \mathrm{Ne} / \mathrm{O}$ b), h), $\log \mathrm{S} / \mathrm{O} \mathbf{c}), \mathbf{i}), \log \mathrm{Cl} / \mathrm{O}$ d), j), $\log \mathrm{Ar} / \mathrm{O}$ e), $\mathbf{k})$ and $\log \mathrm{Fe} / \mathrm{O}$ f), l) vs. oxygen abundance $12+\log \mathrm{O} / \mathrm{H}$ for the emission-line galaxies. Large filled circles are galaxies from the HeBCD sample, dots are galaxies from the SDSS sample. Abundances in the left panel are calculated with the expressions used by Izotov et al. (1994, 1997) and Thuan et al. (1995) and those in the right panel are obtained with expressions from this paper. The solar values as compiled by Lodders (2003) are indicated by the large open circles and the associated error bars are shown. Straight lines are linear regressions obtained for the HeBCD sample.
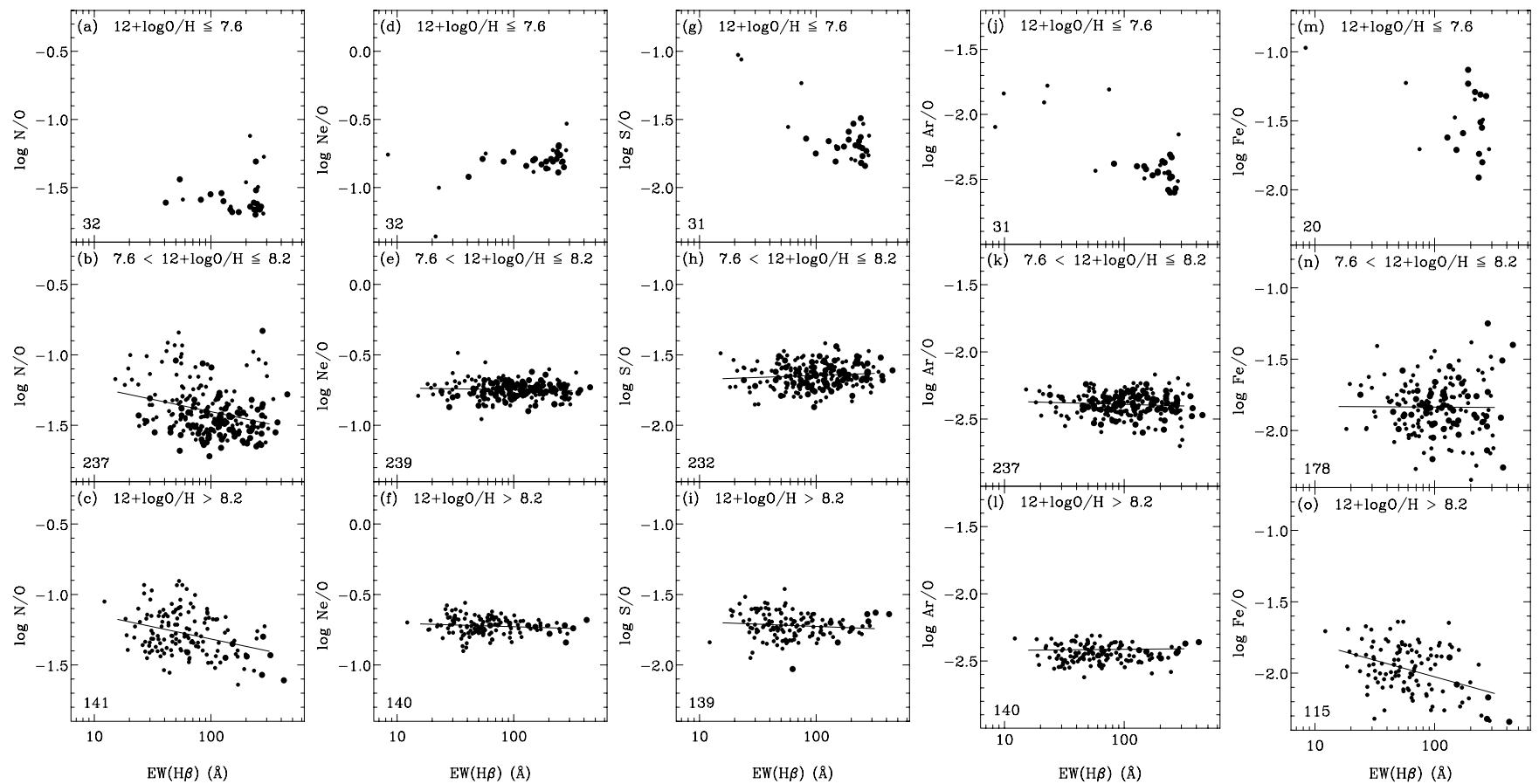

Fig. 12. a)-c) Nitrogen-to-oxygen abundance ratio, $\log \mathrm{N} / \mathrm{O}$, vs. equivalent width of $\mathrm{H} \beta$ emission line, $E W(\mathrm{H} \beta)$, for the galaxies in three metallicity bins. d)-f) The same as in a)-c), but for neon-to-oxygen abundance ratio. g)-i) The same as in a)-c), but for sulfur-to-oxygen abundance ratio. j)-l) The same as in a)-c), but for argon-to-oxygen abundance ratio. m)-o) The same as in a)-c), but for iron-to-oxygen abundance ratio. Straight lines are linear regressions obtained for intermediate $Z$ and high $Z$ samples. 
Table 3. Comparison of element abundances in the galaxies present in both HeBCD and SDSS samples

\begin{tabular}{|c|c|c|}
\hline Name & $(12+\log \mathrm{O} / \mathrm{H})_{\mathrm{SDSS}}$ & $(12+\log \mathrm{O} / \mathrm{H})_{\mathrm{HeBCD}}$ \\
\hline CGCG007-025 & $7.84 \pm 0.02$ & $7.78 \pm 0.01$ \\
\hline HS0822+3542 & $7.42 \pm 0.03$ & $7.45 \pm 0.01$ \\
\hline \multirow[t]{2}{*}{ HS0837+4717 } & $7.63 \pm 0.03$ & $7.61 \pm 0.01$ \\
\hline & $7.60 \pm 0.03$ & \\
\hline HS0924+3821 & $8.15 \pm 0.04$ & $8.09 \pm 0.02$ \\
\hline IZw18NW & $7.14 \pm 0.05$ & $7.16 \pm 0.02$ \\
\hline IZw18SE & $7.25 \pm 0.04$ & $7.19 \pm 0.03$ \\
\hline Mrk35 & $8.29 \pm 0.12$ & $8.37 \pm 0.01$ \\
\hline Mrk178 & $7.90 \pm 0.11$ & $7.82 \pm 0.02$ \\
\hline \multirow[t]{2}{*}{ Mrk1236 } & $7.99 \pm 0.05$ & $8.09 \pm 0.03$ \\
\hline & $8.12 \pm 0.02$ & \\
\hline \multirow[t]{2}{*}{ SBS0917+527 } & $7.81 \pm 0.04$ & $7.87 \pm 0.02$ \\
\hline & $7.91 \pm 0.02$ & \\
\hline SBS0926+606 & $7.99 \pm 0.02$ & $7.92 \pm 0.02$ \\
\hline SBS0946+558 & $8.04 \pm 0.02$ & $8.01 \pm 0.01$ \\
\hline SBS0948+532 & $8.04 \pm 0.03$ & $8.08 \pm 0.01$ \\
\hline SBS1030+583 & $7.79 \pm 0.03$ & $7.80 \pm 0.01$ \\
\hline SBS $1128+573$ & $7.72 \pm 0.05$ & $7.75 \pm 0.03$ \\
\hline SBS1152+579 & $7.95 \pm 0.02$ & $7.88 \pm 0.01$ \\
\hline SBS1205+557 & $7.92 \pm 0.04$ & $7.77 \pm 0.03$ \\
\hline SBS $1211+540$ & $7.65 \pm 0.03$ & $7.64 \pm 0.01$ \\
\hline SBS1222+614 & $8.00 \pm 0.02$ & $7.97 \pm 0.01$ \\
\hline SBS1319+579B & $8.40 \pm 0.10$ & $8.10 \pm 0.01$ \\
\hline SBS1319+579A & $7.75 \pm 0.18$ & $8.17 \pm 0.04$ \\
\hline SBS $1358+576$ & $7.94 \pm 0.03$ & $7.89 \pm 0.02$ \\
\hline SBS1533+574 & $8.11 \pm 0.12$ & $8.14 \pm 0.02$ \\
\hline SBS1533+469 & $8.16 \pm 0.06$ & $7.99 \pm 0.02$ \\
\hline SBS1535+554 & $8.08 \pm 0.02$ & $8.08 \pm 0.04$ \\
\hline UM311 & $8.40 \pm 0.11$ & $8.38 \pm 0.04$ \\
\hline \multirow[t]{2}{*}{ UM461 } & $7.81 \pm 0.02$ & $7.79 \pm 0.03$ \\
\hline & $7.28 \pm 0.04^{1}$ & \\
\hline \multirow[t]{2}{*}{ UM462 } & $7.80 \pm 0.02$ & $7.90 \pm 0.02$ \\
\hline & $7.87 \pm 0.03$ & \\
\hline
\end{tabular}

${ }^{1}[\mathrm{O}$ III] $\lambda 4959,5007$ emission lines are clipped.

indicate that the solar S and Ar abundances are not as accurately known as thought.

We show by solid lines in Fig. 11 (middle and right panels) the regression lines for $\log \mathrm{Ne} / \mathrm{O}, \log \mathrm{S} / \mathrm{O}, \log \mathrm{Cl} / \mathrm{O}, \log$ $\mathrm{Ar} / \mathrm{O}$ and $\log \mathrm{Fe} / \mathrm{O}$ vs. $12+\log \mathrm{O} / \mathrm{H}$ obtained from the HeBCD sample only. The equations of these lines are the following:

$\log \frac{\mathrm{Ne}}{\mathrm{O}}=(0.088 \pm 0.018) X-(1.450 \pm 0.144)$,

$\log \frac{\mathrm{S}}{\mathrm{O}}=-(0.026 \pm 0.027) X-(1.514 \pm 0.215)$,

$\log \frac{\mathrm{Cl}}{\mathrm{O}}=(0.120 \pm 0.106) X-(4.365 \pm 0.852)$,

$\log \frac{\mathrm{Ar}}{\mathrm{O}}=(0.039 \pm 0.031) X-(2.714 \pm 0.244)$,

$\log \frac{\mathrm{Fe}}{\mathrm{O}}=-(0.606 \pm 0.081) X+(2.994 \pm 0.639)$,

where $X=12+\log \mathrm{O} / \mathrm{H}$.
For the merged HeBCD and SDSS sample we find no significant trends with the oxygen abundance for the $\mathrm{S} / \mathrm{O}, \mathrm{Cl} / \mathrm{O}$ and $\mathrm{Ar} / \mathrm{O}$ abundance ratios in the whole range of oxygen abundance (Figs. 11h-k). The best derived abundances among the $\alpha$-elements are for oxygen and neon because the atomic data for these elements are better known, the abundances are derived from bright emission lines and only two ionization stages are present in significant amount in H II regions.

\subsection{Elemental depletion onto dust grains}

We note that there is a slight increase in $\mathrm{Ne} / \mathrm{O}$ with increasing $\mathrm{O} / \mathrm{H}$ in panel h of Fig. 11 (the correlation is significant at the $99.98 \%$ level according to the Spearman statistical test). If the new expressions for deriving abundances are correct then this slight increase (by $\sim 0.1$ dex over the entire metallicity range considered) can be interpreted by depletion of oxygen onto dust grains. This corresponds to $\sim 20 \%$ of oxygen locked in the dust grains in the highest-metallicity $\mathrm{H}$ II regions of our sample, while no significant depletion would be present in the $\mathrm{H}$ II regions with lower metallicity (assuming that the adopted solar $\mathrm{Ne} / \mathrm{O}$ ratio corresponds to that of the interstellar medium). Since $\mathrm{Ar}$ is a noble gas like $\mathrm{Ne}$, one would expect the same trend in $\mathrm{Ar} / \mathrm{O}$ as in $\mathrm{Ne} / \mathrm{O}$. The regression lines for the merged HeBCD and SDSS sample shown in Figs. $11 \mathrm{~h}$ and $\mathrm{k}$ are

$\log \frac{\mathrm{Ne}}{\mathrm{O}}=(0.097 \pm 0.015) X-(1.531 \pm 0.120)$,

$\log \frac{\mathrm{Ar}}{\mathrm{O}}=-(0.084 \pm 0.021) X-(1.729 \pm 0.166)$.

It is seen that the slopes of the $\mathrm{Ne} / \mathrm{O}$ vs. $\mathrm{O} / \mathrm{H}$ and $\mathrm{Ar} / \mathrm{O}$ vs. $\mathrm{O} / \mathrm{H}$ regressions are statistically different. This could indicate that the production of Ar relative to $\mathrm{O}$ by the stellar populations decreases with increasing metallicity.

As seen in Fig. 11, iron has a very different behavior as compared with other elements. The $\mathrm{Fe} / \mathrm{O}$ ratio is well below solar for most of the galaxies. In addition, the trend with $\mathrm{O} / \mathrm{H}$ is opposite to that in metal-poor stars in our Galaxy (Carretta et al. 2000; Takeda 2003; Bai et al. 2004). This argues in favor of depletion of iron onto dust grains. The decrease of the $\mathrm{Fe} / \mathrm{O}$ abundance ratio with increasing $\mathrm{O} / \mathrm{H}$ (by $\sim 1$ dex when the oxygen abundance increases by $\sim 1.5$ dex as seen in Fig. 111) implies that depletion of $\mathrm{Fe}$ increases with increasing metallicity, similar to what is suggested by Rodriguez \& Rubin (2005) from the consideration of a small sample of Galactic and extragalactic $\mathrm{H}$ II regions. If this explanation is correct then only $\$ 10 \%$ of iron in the high-metallicity $\mathrm{H}$ II regions of our sample $(\log \mathrm{O} / \mathrm{H}+12=8.2-8.5)$ is in gas phase and the remaining $\gtrsim 90 \%$ of iron is locked in dust grains, while in the $\mathrm{H}$ II regions with the lowest metallicity the depletion is much lower. This conclusion is supported by the small trend seen in the $\mathrm{Ne} / \mathrm{O}$ abundance ratio. Since Ne is not locked in dust grains, the increase of $\mathrm{Ne} / \mathrm{O}$ with metallicity can be explained by a small depletion of oxygen. Since oxygen is an element $\sim 15$ times more abundant than iron, a depletion of oxygen by $\sim 20 \%$ would correspond to an important iron depletion. In the panels on the right of Fig. 12, we show the variation of $\mathrm{Fe} / \mathrm{O}$ as a function 
of the equivalent width of $\mathrm{H} \beta, E W(\mathrm{H} \beta)$, for the galaxies in the SDSS and HeBCD merged sample. Figure $12 \mathrm{~m}$ corresponds to the lowest metallicity bin, defined by $12+\log \mathrm{O} / \mathrm{H} \leq 7.6$, Fig. $12 \mathrm{n}$ corresponds to the intermediate metallicity bin $(7.6<$ $12+\log \mathrm{O} / \mathrm{H} \leq 8.2)$ and Fig. 12o corresponds to the highest metallicity bin in our sample $(12+\log \mathrm{O} / \mathrm{H}>8.2)$. If we take $E W(\mathrm{H} \beta)$ as a measure of the age of the ionizing stars (but see Stasińska \& Izotov 2003), Fig. 12o strongly suggests that the grains are gradually destroyed on a time scale of several Myr. The Spearman rank correlation coefficient is $R_{\mathrm{S}}=-0.30$ with an associated significance level of $99.9 \%$, indicating that the observed correlation is not due to chance. There is no similar clear trend in Fig. 12n $\left(R_{\mathrm{S}}=-0.006\right.$ with an associated significance level of $7 \%$ ). However, since the initial depletion in the intermediate metallicity bin is lower than in the highest metallicity bin, detecting a temporal variation of the correlation is more difficult. Note that the scatter in $\mathrm{Fe} / \mathrm{H}$ at any value of $E W(\mathrm{H} \beta)$ as well as at any value of $\mathrm{O} / \mathrm{H}$ is far more important in $\mathrm{Fe} / \mathrm{O}$ than in $\mathrm{Ne} / \mathrm{O}, \mathrm{S} / \mathrm{O}, \mathrm{Cl} / \mathrm{O}$ and $\mathrm{Ar} / \mathrm{O}$. We believe that this is mostly due to errors in the iron abundance determinations since the $[\mathrm{Fe}$ III] lines are generally very weak but one cannot exclude that the degree of iron depletion might vary among $\mathrm{H}$ II regions of similar characteristics.

\subsection{The nitrogen conundrum}

Much has already been written about the N/O versus $\mathrm{O} / \mathrm{H}$ diagram (upper line of panels in Fig. 11) (e.g., Garnett 1990; Pilyugin 1993; Henry et al. 2000). Our new results basically confirm the findings in our previous papers, based on considerably smaller samples (Izotov \& Thuan 1999; Izotov et al. 2004): the $\mathrm{N} / \mathrm{O}$ ratio decreases as $\mathrm{O} / \mathrm{H}$ decreases but reaches a plateau at $12+\log \mathrm{O} / \mathrm{H} \leq 7.6$. Moreover, while at a given $\mathrm{O} / \mathrm{H}$, there is a significant dispersion in $\mathrm{N} / \mathrm{O}$ when $12+\log \mathrm{O} / \mathrm{H}>$ 7.6, the dispersion is very small at lower values of $\mathrm{O} / \mathrm{H}$. The lack of $\mathrm{H}$ II regions with $12+\log \mathrm{O} / \mathrm{H} \leq 7.6$ and high $\mathrm{N} / \mathrm{O}$ is likely not due to selection effects. Indeed higher N/O abundance ratio implies higher flux of the [N II] $\lambda 6584$ emission line which can be more easily detected in this case. On the other hand, it is unlikely that we missed low-metallicity galaxies with a very low $\mathrm{N} / \mathrm{O}$ abundance ratio, below the plateau value of $\log$ $\mathrm{N} / \mathrm{O} \sim-1.6$, as the $[\mathrm{N}$ II] $\lambda 6584$ emission line was detected in all most metal-deficient galaxies.

The N/O versus $\mathrm{O} / \mathrm{H}$ pattern for $\mathrm{H}$ II regions differs significantly from that for damped Ly $\alpha$ systems (DLA) (e.g., Centurión et al. 2003; Molaro 2005), in that some DLAs have $\mathrm{N} / \mathrm{O}$ ratios much lower than $-1.6 \mathrm{dex}$, the value of the observed plateau for $\mathrm{H}$ II regions. Recently, the $\mathrm{N} / \mathrm{O}-\mathrm{O} / \mathrm{H}$ diagram has also been constructed for metal-poor stars (Spite et al. 2005). Stars affected by mixing have been excluded from their diagram. It is seen that some very low-metallicity stars also have values of N/O below the $\mathrm{H}$ II region plateau, although they are larger than the lowest N/O found for DLAs. To interpret these results in terms of models of chemical evolution of galaxies, we should however bear in mind that abundances determined in DLAs (see Dessauges-Zavadsky et al. 2004) as well as in stars (Spite et al. 2005) are generally less accurate than the
$\mathrm{N} / \mathrm{O}$ ratios in metal-poor $\mathrm{H}$ II regions where the main source of uncertainty is the measurement of the weak [N II] 6584 line. In the HeBCD sample, the average formal error in N/O is $\lesssim 0.1$ dex at $12+\log \mathrm{O} / \mathrm{H} \leq 7.7$, and it is $\leq 0.2$ dex for bright galaxies in the SDSS DR3 sample at the same metallicities. Also, DLAs, Galactic halo stars and metal-poor emission-line galaxies had likely very different star formation histories. The differences in the abundance patterns observed between these systems might also result from the different timescales and intensities of the star formation episodes which enriched their interstellar medium.

The fact that no $\mathrm{H}$ II region is found with an extremely low N/O abundance ratio $(\log \mathrm{N} / \mathrm{O} \leq-1.6)$ suggests that the SDSS galaxies are not extremely young and have ages more than 100-300 Myr, which are required for the completion of the evolution of the intermediate-mass stars.

We also confirm the trend of increasing N/O with decreasing $E W(\mathrm{H} \beta)$ already mentioned by Izotov et al. (2004). This trend is seen in the $12+\log \mathrm{O} / \mathrm{H}>8.2$ metallicity bin (at a 99.99\% significance level according to the Spearman statistics) and also in the $7.6<12+\log \mathrm{O} / \mathrm{H}<8.2$ metallicity bin (at a 99.8\% significance level) (panels c and b of Fig. 12). Note that, apart from $\mathrm{Fe} / \mathrm{O}$, which we discussed before, none of the remaining abundance ratios shown in Fig. 12 exhibits such a trend. Therefore, the trend for $\mathrm{N} / \mathrm{O}$ with $E W(\mathrm{H} \beta)$ must be real and not due to selection effects or systematic errors in abundance determinations.

Since $E W(\mathrm{H} \beta)$ is a measure of the age of the ionizing-star population, it is interesting to check whether the larger N/O abundance ratio in the more evolved starbursts is due to the nitrogen enrichment by the Wolf-Rayet stars.

Let us consider the massive star rotating models for a metallicity equal to $Z=0.004$, which corresponds to $12+\log \mathrm{O} / \mathrm{H}=$ 8.2 , computed recently by Meynet \& Maeder (2005). The $60 M_{\odot}$ star model loses about half of its initial mass during the first 4.76 Myr. During this phase the amount of nitrogen in the wind ejected material is about 9 times the initial content (the initial content would be the mass of nitrogen ejected if no change of nitrogen abundance occurred). On the other hand, oxygen appears to be depleted by a factor 2.5 with respect to its initial value. Thus, we conclude that if one were to observe only wind material of this kind then one would expect an increase of the N/O ratio by about a factor 20 in a few Myrs. A similar estimate made on the basis of a $120 M_{\odot}$ stellar model leads to an N/O increase by a factor 55 , while a $30 M_{\odot}$ stellar model would predict an increase of only a factor 5 . Of course, there is a dilution of the WR material with the local interstellar material of the $\mathrm{H}$ II region ${ }^{2}$. The enhancement factor of the N/O abundance ratio in the $\mathrm{H}$ II region due to the WR wind can be estimated as

$\frac{\mathrm{N} / \mathrm{O}(\mathrm{HII})}{\mathrm{N} / \mathrm{O}(\mathrm{ini})}=\frac{p(\mathrm{~N}) \times\left(M_{\mathrm{w}} / M_{*}\right) \times\left(M_{*} / M(\mathrm{HII})\right)+1}{p(\mathrm{O}) \times\left(M_{\mathrm{w}} / M_{*}\right) \times\left(M_{*} / M(\mathrm{HII})\right)+1}$,

\footnotetext{
${ }^{2}$ Note also that these stars may also eject through their winds and at the time of the supernova explosion material with very low N/O ratios. This would also concur, together with the dilution effect, to decrease the $\mathrm{N} / \mathrm{O}$ ratio in the $\mathrm{H}$ II region.
} 
where $p(\mathrm{~N})$ and $p(\mathrm{O})$ are the production factors of nitrogen and oxygen in the wind, $M_{\mathrm{w}}$ is the total mass in the winds, $M_{*}$ is the integrated initial mass of the stars and $M(\mathrm{H}$ II $)$ is the mass of the $\mathrm{H}$ II region. Let us take $p(\mathrm{~N})=9, p(\mathrm{O})=0.4$, typical of the $60 M_{\odot}$ model. In the mass range between 30 and $120 M_{\odot}$, on average about half of the stellar mass is lost through stellar winds enriched in $\mathrm{N}$ and depleted in $\mathrm{O}$, thus

$$
M_{\mathrm{w}} / M_{*} \approx \frac{\int_{30}^{120} 0.5 M M^{-2.35} \mathrm{~d} M}{\int_{0.1}^{120} M M^{-2.35} \mathrm{~d} M} \sim 0.03 .
$$

About 3 percent of the mass in stars is ejected under the form of N-rich stellar wind. For an $\mathrm{H}$ II region of constant number density $n$, the value of $M_{*} / M(\mathrm{H}$ II) for a cluster with a Salpeter initial mass function is roughly equal to $n / 360$. If the number density of the $\mathrm{H}$ II region is $100 \mathrm{~cm}^{-3}, M_{*} / M(\mathrm{H} \mathrm{II})$ is about 0.28 . Thus the enhancement factor of the $\mathrm{N} / \mathrm{O}$ abundance ratio in the $\mathrm{H}$ II region is $\sim 1.07$ ( or $\sim 0.03 \mathrm{dex}$ ), significantly lower than the dispersion of the N/O abundance ratio in Fig. 11 and the value of the trend seen in Fig. 12 (left panel).

It is likely, therefore, that the large dispersion of N/O abundance ratio in Fig. 11 and the trend seen in Fig. 12 are not due to a global increase of the N/O ratio in the $\mathrm{H}$ II region, but it could still be a local effect. It was assumed in the above consideration that the $\mathrm{H}$ II region is uniform, i.e. its density is constant. However, it is very likely that the density of the $\mathrm{N}$ enriched ejecta is much higher than that in the ambient $\mathrm{H}$ II region. Since the luminosity of the forbidden lines in the lowdensity medium scales as the number density of the emitting ions multiplied by the number density of electrons, the apparent enhancement of the N/O abundance ratio, can be estimated from

$$
\left[\frac{\mathrm{N} / \mathrm{O}(\mathrm{HII})}{\mathrm{N} / \mathrm{O}(\text { ini })}\right]_{\mathrm{app}}=\frac{p(\mathrm{~N}) \times n_{\mathrm{ej}} / n \times M_{\mathrm{w}} / M(\mathrm{HII})+1}{p(\mathrm{O}) \times n_{\mathrm{ej}} / n \times M_{\mathrm{w}} / M(\mathrm{HII})+1},
$$

where $n_{\mathrm{ej}}$ is the number density of gas particles in the ejecta. Adopting $n=100 \mathrm{~cm}^{-3}$ and $n_{\mathrm{ej}}=1000 \mathrm{~cm}^{-3}$ we obtain an apparent enhancement factor of $\sim 1.7$ from Eq. (34), comparable to the dispersion of the N/O abundance ratio in Fig. 11 and the value of the trend in Fig. 12. Note that there is no direct diagnostic of the density of the emitting ejecta. The density derived from the $[\mathrm{S} \mathrm{II}]$ line ratio applies essentially to the $\mathrm{H}$ II since the winds are not enhanced in sulfur.

In summary, we find that the observed trend of N/O increasing as $E W(\mathrm{H} \beta)$ decreases is naturally explained by the expected ejection from Wolf-Rayet stars. However, the true value of the N/O enhancement factor is defined by Eq. (32), and is small. The dispersion of the observed N/O abundance ratio is caused by the local enrichment by the WR stars and the value of the N/O abundance ratio in the $\mathrm{H}$ II before enrichment by the present starburst is likely defined by the lower boundary in the $\mathrm{N} / \mathrm{O}$ vs. $\mathrm{O} / \mathrm{H}$ diagram. If this is correct then the small dispersion of the $\mathrm{N} / \mathrm{O}$ abundance ratio in the most-metal deficient $\mathrm{H}$ II regions could perhaps be explained by the very low number of the WR stars and short WR stage during the low-metallicity burst of star formation. However, a larger sample of extremely low-metallicity objects would be needed to confirm this.

\section{Conclusions}

We have examined all the galaxies in the Sloan Digital Sky Survey (SDSS) Data Release 3 (DR3) to select out those with a detected [O III] $\lambda 4363$ emission line, which allows, in principle, a direct element abundance determination based on the electron temperature. To minimize errors in the abundance determinations, we have kept for further analysis only $\sim 310$ SDSS DR3 galaxies that are sufficiently bright in $\mathrm{H} \beta$ $\left(F(\mathrm{H} \beta)>10^{-14} \mathrm{erg} \mathrm{s}^{-1} \mathrm{~cm}^{-2}\right)$. The [O III] $\lambda 4363$ line in these objects is detected at the $\gtrsim 2 \sigma$ level. This sample was merged with a sample of 109 blue compact dwarf galaxies with highquality observations and large equivalent widths of emission lines, referred to as the HeBCD sample. In this way we obtained samples of emission-line galaxies, spanning a range in metallicities from $12+\log \mathrm{O} / \mathrm{H} \sim 7.1\left(Z_{\odot} / 30\right)$ to $\sim 8.5\left(0.7 Z_{\odot}\right)$.

Our main conclusions are as follows:

1. Despite an examination of the entire SDSS DR3 sample of $\sim 530000$ galaxies, we found only 6 new galaxies with extremely low metallicity $(12+\log \mathrm{O} / \mathrm{H}<7.6$, i.e. $Z<Z_{\odot} / 12$ ), in addition to the galaxies discovered by Kniazev et al. (2003). For comparison, the HeBCD sample which consists of only $\sim 100$ galaxies contains about 15 such galaxies. This difference is in part due to selection effects. The selection criteria for the HeBCD sample favour extremely low-metallicity galaxies as they were collected for the determination of the primordial He abundance. The small number of extremely low-metallicity galaxies in the SDSS may suggest that such galaxies are actually very rare. However, the selection effects in the SDSS might be important because most metal-poor galaxies are in general lowluminosity objects and the SDSS spectroscopic data are only complete for galaxies brighter than $\sim 17.7 \mathrm{mag}$ (Izotov et al. 2004).

2. The emission-line galaxies in the SDSS sample show distributions of the N/O, Ne/O, S/O, Cl/O, Ar/O, Fe/O abundance ratios vs. oxygen abundance similar to those found earlier for the galaxies in the HeBCD sample (Thuan et al. 1995; Izotov \& Thuan 1999; Guseva et al. 2003).

3. The $\alpha$ element-to-oxygen abundance ratios $\mathrm{Ne} / \mathrm{O}, \mathrm{Cl} / \mathrm{O}$, $\mathrm{S} / \mathrm{O}$ and $\mathrm{Ar} / \mathrm{O}$ do not show large trends with oxygen abundance. The best determined ratio, $\mathrm{Ne} / \mathrm{O}$, increases slightly with increasing $\mathrm{O} / \mathrm{H}$ (by $\sim 0.1$ dex in the considered metallicity range). This can be explained by the depletion of oxygen onto dust grains with $\sim 20 \%$ of O locked in dust. No significant depletion of oxygen is found in the lowermetallicity $\mathrm{H}$ II regions. The mean $\mathrm{Ne} / \mathrm{O}$ and $\mathrm{Cl} / \mathrm{O}$ abundance ratios in the $\mathrm{H}$ II regions from our sample are consistent with the solar ratios. On the other hand, the mean S/O and $\mathrm{Ar} / \mathrm{O}$ abundance ratios in the $\mathrm{H}$ II regions are lower than the solar ratio. This may indicate the $\mathrm{S}$ and $\mathrm{Ar}$ abundances in the Sun are somewhat uncertain.

4. The $\mathrm{Fe} / \mathrm{O}$ abundance ratio shows an underabundance of iron relative to oxygen as compared to solar, suggesting depletion of iron onto dust grains. In the high-metallicity galaxies more than $90 \%$ of iron is locked onto dust grains. On the other hand, no significant iron depletion is present in 
the lowest-metallicity galaxies. We also find evidence that dust destruction occurs on a time scale of several Myr.

5. No galaxy with $\log \mathrm{N} / \mathrm{O} \lesssim-1.6$ was found, suggesting that the chemical evolution of local low-metallicity emissionline galaxies is different from that of some high-redshift DLAs which have considerably lower log N/O ( - 2.3). These DLAs are considered to be truly young galaxies, with nitrogen produced only by massive stars. If this interpretation is correct, then our sample of $\sim 400$ dwarf emissionline galaxies contains no extremely young galaxy but rather galaxies of ages $\gtrsim 100-300 \mathrm{Myr}$, required for the enrichment in nitrogen by intermediate-mass stars.

6. Our data indicate an apparent increase of N/O with decreasing $E W(\mathrm{H} \beta)$, best seen among galaxies from our sample that have intermediate metallicities. We interpret this as evidence for gradual enrichment of the $\mathrm{H}$ II region in nitrogen by massive stars from the most recent starburst. The magnitude of the observed effect is consistent with that expected from current theoretical models for massive stars, if account is taken for the density of the ejecta being larger than that of the $\mathrm{H}$ II regions.

Acknowledgements. Y.I.I. acknowledges the support of the Observatoire de Paris, and Y.I.I. and N.G.G. thank the hospitality of the Observatoire de Genève where part of this work was carried out. Y.I.I., G.M. and N.G.G. acknowledge the support of the Swiss SCOPE 7UKPJ62178 grant. T.X.T. and Y.I.I. acknowledge the partial financial support of NSF grant AST 02-05785. The research described in this publication was made possible in part by Award No. UP1-2551-KV-03 of the US Civilian Research \& Development Foundation for the Independent States of the Former Soviet Union (CRDF). All the authors acknowledge the work of the Sloan Digital Sky Survey (SDSS) team. Funding for the SDSS has been provided by the Alfred P. Sloan Foundation, the Participating Institutions, the National Aeronautics and Space Administration, the National Science Foundation, the US Department of Energy, the Japanese Monbukagakusho, and the Max Planck Society. The SDSS Web site is http://www.sdss.org/. The SDSS is managed by the Astrophysical Research Consortium (ARC) for the Participating Institutions. The Participating Institutions are The University of Chicago, Fermilab, the Institute for Advanced Study, the Japan Participation Group, The Johns Hopkins University, the Korean Scientist Group, Los Alamos National Laboratory, the Max-PlanckInstitute for Astronomy (MPIA), the Max-Planck-Institute for Astrophysics (MPA), New Mexico State University, University of Pittsburgh, University of Portsmouth, Princeton University, the United States Naval Observatory, and the University of Washington.

\section{References}

Abazajian, K., Adelman-McCarthy, J. K., Agüeros, M. A., et al. 2005, AJ, 129, 1755

Aller, L. H. 1984, Physics of Thermal Gaseous Nebulae (Dordrecht: Reidel) Geochim. Cosmochim. Acta, 53, 197

Bai, G. S., Zhao, G., Chen, Y. Q., et al. 2004, A\&A, 425, 671

Carretta, E., Gratton, R. G., \& Sneden, C. 2000, A\&A, 356, 238

Centurión, M., Molaro, P., Vladilo, G., et al. 2003, A\&A, 403, 55

Dessauges-Zavadsky, M., Calura, F., Prochaska, J. X., D’Odorico, S., \& Matteucci, F. 2004, A\&A, 416, 79

Fillipenko, A. V. 1982, PASP, 94, 715

Garnett, D. R. 1990, ApJ, 363, 142

Garnett, D. R. 1992, AJ, 103, 1330

Guseva, N. G., Papaderos, P., Izotov, Y. I., et al. 2003c, A\&A, 407, 105

Henry, R. B. C., Edmunds, M. G., \& Köppen, J. 2000, ApJ, 541, 660

Izotov, Y. I., \& Thuan, T. X. 1999, ApJ, 511, 639

Izotov, Y. I., \& Thuan, T. X. 2004a, ApJ, 602, 200

Izotov, Y. I., \& Thuan, T. X. 2004b, ApJ, 616, 768

Izotov, Y. I., Thuan, T. X., \& Lipovetsky, V. A. 1994, ApJ, 435, 647

Izotov, Y. I., Thuan, T. X., \& Lipovetsky, V. A. 1997, ApJS, 108, 1

Izotov, Y. I., Stasińska, G., Guseva, N. G., \& Thuan, T. X. 2004, A\&A, 415,87

Kauffmann, G., Heckman, T. M., Tremonti, C., et al. 2003, MNRAS, 346, 1055

Kniazev, A. Y., Grebel, E. K., Hao, L., et al. 2003, ApJ, 593, 73

Kniazev, A. Y., Pustilnik, S. A., Grebel, E. K., Lee, H., \& Pramskij, A. G. 2004, ApJS, 153, 429

Leitherer, C., Schaerer, D., Goldader, J. D., et al. 1999, ApJS, 123, 3

Lodders, K. 2003, ApJ, 591, 1220

Meynet, G., \& Maeder, A. 2005, A\&A, 429, 613

Molaro, P. 2005, preprint [arXiv: astro-ph/0503214]

Oke, J. B. 1990, AJ, 99, 1621

Pilyugin, L. S. 1992, A\&A, 260, 58

Ramsbottom, C. A., Bell, K. L., \& Stafford, R. P. 1996, ADNDT, 63, 57

Rodriguez, M., \& Rubin, R. H. 2005, ApJ, 626, 900

Smith, L. J., Norris, R. P. F., \& Crowther, P. A., 2002, MNRAS, 337, 1309

Spite, M., Cayrel, R., Plez, B., et al. 2005, A\&A, 430, 655

Stasińska, G. 1990, A\&AS, 83, 501

Stasińska, G. 2005, A\&A, 434, 507

Stasińska, G., \& Izotov, Y. I. 2003, A\&A, 397, 71

Takeda, Y. 2003, A\&A, 402, 343

Thuan, T. X., Izotov, Y. I., \& Lipovetsky, V. A. 1995, ApJ, 445, 108

York, D. G., Adelman, J., Anderson, J. E., Jr., et al. 2000, AJ, 120, 1579

Whitford, A. E. 1958, AJ, 63, 201 